\title{
Glial alterations in tuberculous and cryptococcal meningitis and their relation to HIV co-infection - A study on human brains
}

\author{
Surya Tripathi ${ }^{1,2}$, Ishan Patro ${ }^{2}$, Anita Mahadevan ${ }^{1}$, Nisha Patro ${ }^{2}$, Mariamma Phillip ${ }^{3}$, Susarla Krishna \\ Shankar ${ }^{1}$ \\ ${ }^{1}$ Department of Neuropathology, National Institute of Mental Health and Neuro Sciences, Bangalore, India \\ ${ }^{2}$ School of Studies in Neuroscience, Jiwaji University, Gwalior, India \\ ${ }^{3}$ Department of Biostatistics, National Institute of Mental Health and Neuro Sciences, Bangalore, India
}

\begin{abstract}
Introduction: Tuberculosis and cryptococcal infection of the central nervous system are common AIDS-associated opportunistic infections in tropical underdeveloped and developing countries. To date, research on these infections has focused on clinical, imaging, laboratory diagnosis, and animal models to elucidate the pathogenesis. There is paucity of information on astroglial and microglial alterations in the human nervous system following these infections.

Methodology: The pathomorphologic and morphometric alterations of astroglia and microglia in the prefrontal cortex and hippocampus in cases of tuberculous meningitis (TBM) and cryptococcal meningitis (CM) with and without associated HIV were described and compared with cases of HIV encephalitis without opportunistic infections (OI) and HIV-negative human brain tissue.

Results: In TBM, the microglia and astrocytes were activated with hypertrophy and hyperplasia, aggregating in the subpial zone and around granulomas in meningeal exudate. In cases of cryptococcal meningitis, reactive changes were less prominent, though activation of both cellular elements was found. Association of HIV with these OIs resulted in muted glial and microglial response. In HIV encephalitis without OI, the level of activation of was low. Both astroglial and microglial cells expressed caspase-3, a pro-apoptotic marker, following HIV and opportunistic infections. Neuronal apoptosis, a mechanism to ensure neuronal survival, was less evident. The reactive astrocytes and microglia following opportunistic infection developed dystrophic changes heralding senescence.

Conclusions: Further studies on neuronal-astroglial-microglial interaction will offer deeper insight into the pathogenetic and immune mechanisms in the cellular and pathomorphological evolution of tuberculous and cryptococcal infections.
\end{abstract}

Key words: tuberculous meningitis; cryptococcal meningitis; astroglia; microglia; opportunistic infections

J Infect Dev Ctries 2014; 8(11):1421-1443. doi:10.3855/jidc.3894

(Received 19 June 2013 - Accepted 13 May 2014)

Copyright (c) 2014 Tripathi et al. This is an open-access article distributed under the Creative Commons Attribution License, which permits unrestricted use, distribution, and reproduction in any medium, provided the original work is properly cited.

\section{Introduction}

HIV infection causes significant morbidity and mortality. In 2009, 33.3 million adults and children were reported to be living with HIV, nearly two-thirds of them in sub-Saharan Africa [1]. About 2.6 million individuals were newly infected, with the majority of them in low-income and middle-income countries [2]. Among various opportunistic bacterial, viral, and neoplastic AIDS-defining illnesses, neurotuberculosis, cryptococcal meningitis, and toxoplasma encephalitis predominate due to endemicity, leading to high morbidity and mortality. The introduction of combination antiretroviral therapy in 1996 has greatly reduced the incidence of immune deficiency associated opportunistic infections from 13.1/1,000 patients in 1996-1997 to $1.0 / 1,000$ patients in 2006$2007[3,4]$.
The World Health Organization (WHO) estimates that nearly one-third of the world's population is infected with Mycobacterium tuberculosis; HIV coinfected individuals are at a greater risk of acquiring the disseminated active form of the disease, including tuberculous meningitis [5]. In developing countries, HIV-associated opportunistic infection by Cryptococcus neoformans, an encapsulated yeastcausing meningitis, competes with tuberculous meningitis in prevalence. Exposure to both Mycobacterium tuberculosis and Cryptococcus occurs via inhalation, leading to primary pulmonary infection, latent infection, and dissemination of the disease following reduced immune status of the host. While tuberculous meningitis (TBM) is considered to be secondary to activation of Rich's focus in the leptomeninges and choroid plexus [6], cryptococcal 
meningitis $(\mathrm{CM})$ is secondary to reactivation of the latent infection in the lungs of immunocompromised patients [7]. Both the organisms result in meningitis with variable inflammatory responses and parenchymal lesions of diverse morphology.

In our earlier study, inflammatory cells, especially the macrophages, facilitated the entry of HIV into the brain, crossing the blood-brain barrier, in both mycobacterial and cryptococcal meningitc processes [8]. In addition, HIV-infected cells were present in the choroid plexus in both of these infective conditions, reflecting the probable route of entry. Recovery of the virus from the brain tissue and cerebrospinal fluid (CSF) provided evidence for the role of HIV in causing primary infection of the brain in human subjects, forming the basis for evolution of HIVassociated cognitive deficits and HIV-associated dementia, with possible synergic participation of inflammatory cellular elements in the opportunistic chronic meningitic process [9]. Participation of migrating hematogenous macrophages, native brain microglia and astrocytes in the chemokine- and cytokine-mediated inflammatory process has been suggested in the evolution of the pathology based on animal models and in vitro studies [10]. Human studies to date essentially have addressed mostly the clinical, laboratory parameters, and neuroimaging features [11-13]. The earlier neuropathological studies addressed mostly the gross neuroanatomical pathology and histopathological aspects, relegating the cellular pathology of astroglia and microglial elements to the margins. To address this knowledge gap, this study examined astroglial and microglial cellular pathology in cases of TBM and cryptococcal meningitis with and without associated HIV infection and contrasted it with glial response in HIV-infected and relatively normal control adult brains not associated with the meningitic process. Two neuroanatomical areas affected by the meningitic process and involved in neurocognitive pathology, the superior and middle frontal gyri in the prefrontal area and the hippocampus, were chosen for evaluating the astroglial and microglial response and their activation status due to infection by immunohistochemistry.

\section{Methodology}

Human brain tissue from patients with CNS infections (tuberculous and cryptococcal meningitis, five cases each with and without HIV) were examined (Table 1). Archived paraffin blocks from the frontal cortex and hippocampus (either side, with exudate) from confirmed cases of tuberculous meningitis and cryptococcal meningitis, with or without associated HIV and cases of HIV encephalitis without associated opportunistic infections (five cases each) were sourced from the Human Brain Tissue Repository, Department of Neuropathology, National Institute of Mental Health and Neuro Sciences, Bangalore, South India. The clinical autopsies of these cases (confined to examination of the brain only) were conducted 6-12 hours postmortem, after written informed consent of close relatives to utilize the brain tissue for research was obtained. Confidentiality was maintained. The Institutional Scientific Ethics Committee approved the study on the archival pathological material. For noninfectious controls, paraffin blocks from the same neuroanatomical areas from adult victims of road traffic accidents (short stay in the Intensive Care Unit maintaining oxygen saturation, fluid electrolyte balance prior to death, no grossly visible injury, short postmortem interval between the time of death and autopsy, no histologically evident pathology at the anatomical sites, HIV negative by the India National AIDS Control Organization guidelines on testing the antemortem/postmortem blood samples) were selected and included in the study.

\section{Criteria for definitive diagnosis of the infective conditions}

The diagnosis of tuberculous meningitis was based on one or more of the following criteria:

(a) postmortem brain had basal exudates of variable thickness enmeshing the cranial nerves, blood vessels, with or without arterial infarcts in the basal ganglia, with or without hydrocephalus, and histology revealed granulomatous pathology with vasculitis; (b) detection of acid-fast bacilli in the basal exudates/CSF by Ziehl-Neelsen stain; and; (c) detection of antimycobacterial antibody and anti-MTB IgM immune complexes in CSF and serum by enzymelinked immunosorbent assay (ELISA).

The diagnosis of HIV-1 was established by serological and other tests prescribed by the National AIDS Control Organization, India, for definitive diagnosis. Postmortem blood CD4 count by FACS and HIV viral load by nucleic acid sequence based amplification (NASBA) were estimated; HIV -1 clade $\mathrm{C}$ status was determined by clade $\mathrm{C}$-specific polymerase chain reaction (PCR) [14] on plasma or CSF. 
Table 1. Clinical and pathological details of the cases

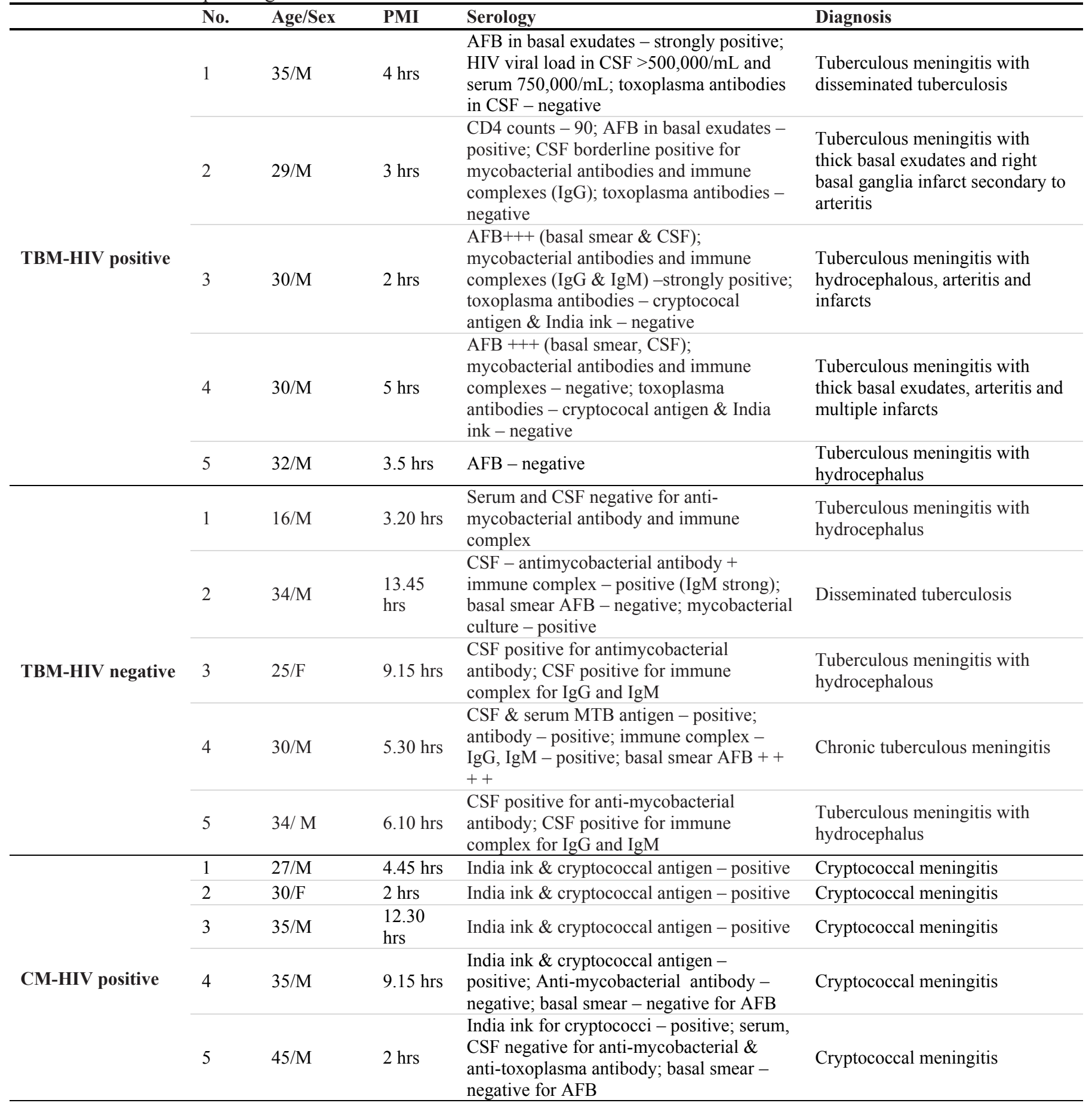


Table 1 (continued). Clinical and pathological details of the cases

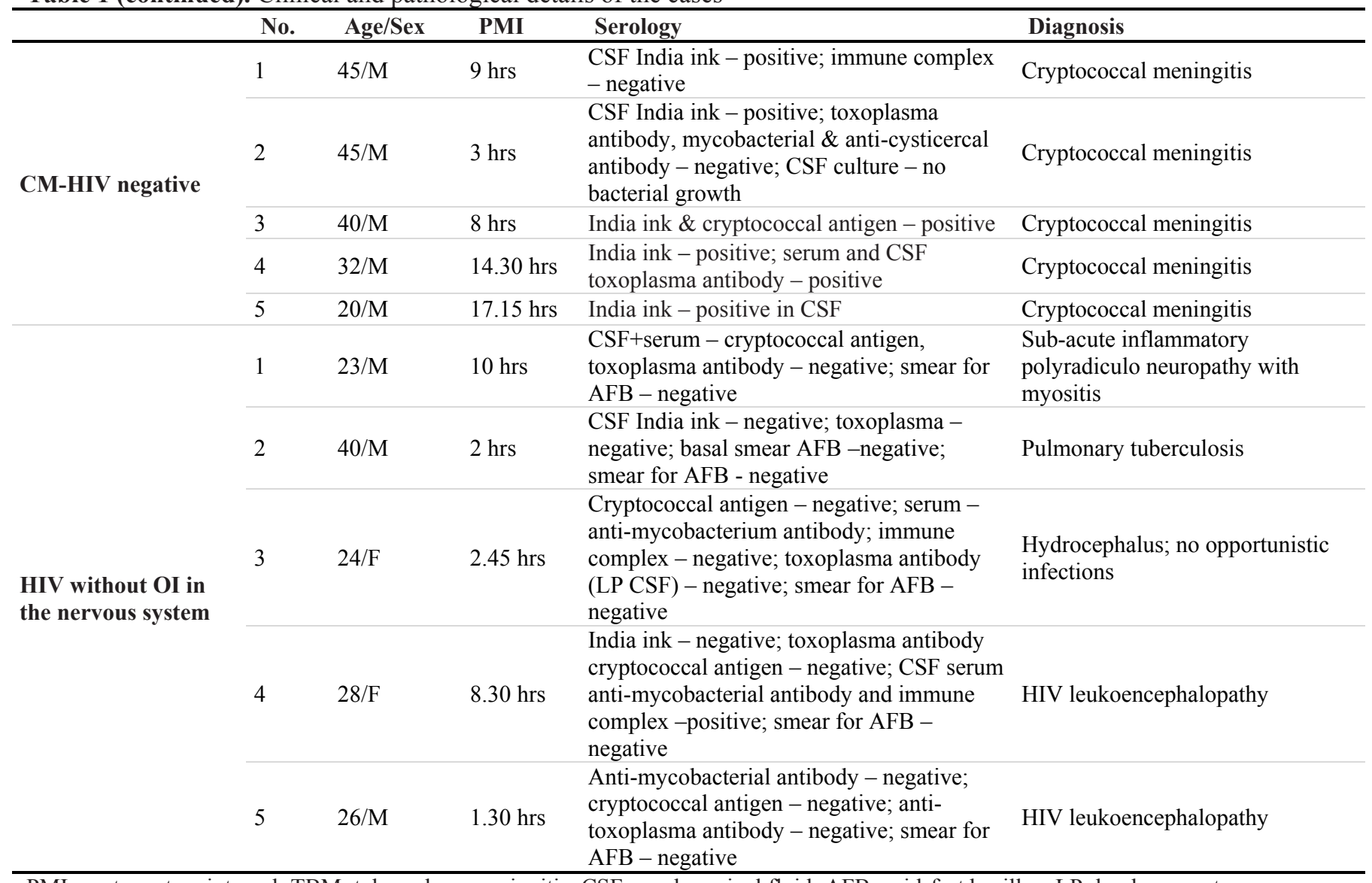

PMI: post-mortem interval; TBM: tuberculous meningitis; CSF: cerebrospinal fluid; AFB: acid-fast bacillus; LP: lumbar puncture

Cryptococcal meningitis was diagnosed based on one or more of the following criteria:

(a) CSF was positive for cryptococci by India ink preparation; (b) cryptococcal antigen was demonstrated in CSF by a latex agglutination test; (c) gross examination of the brain revealed mucoid exudate on the surface and the cut surface had small cystic spaces in cortical ribbon, basal ganglia, diencephalic nuclei and/or brain stem; and (d) histological examination of the sections revealed periodic acid-Schiff (PAS)-positive, mucicarminepositive, capsulated, budding yeast forms in the subarachnoid space and along the Virchow-Robin space.

Serum and CSF samples were tested by ELISA for IgG and IgM antibodies to Toxoplasma gondii and the selected brains were diligently examined to exclude associated toxoplasma encephalitis.

The cases selected as HIV positive without associated opportunistic mycobacterial or cryptococcal infection were serologically and histopathologically negative for these two pathogens in addition to toxoplasmosis and common viral infections.

\section{Immunohistochemical analysis}

Immunohistochemistry for the study of astrocytes, microglial and macrophage lineage of cells was carried out by standard indirect immunoperoxidase technique using the streptavidin-biotin horseradish peroxidase (HRP) method (Table 2).

Serial sections from the two anatomical sites (superior frontal gyrus and hippocampus) were stained with hematoxylin and eosin for routine histological evaluation and with luxol fast blue for assessing myelin pathology.

Quantitative assessment of astrocytic and microglial cell response in different infections and association with HIV

Three paraffin sections of 5 micron thickness at the intervals of $25 \mu \mathrm{m}$ were collected from both the 
frontal cortex and hippocampus from all the samples for the quantitative assessment.

Images from glial fibrillary acidic protein (GFAP) and ionized calcium binding adaptor molecule 1 (Iba1)-immunolabeled slides were captured by a digital camera (DC-200) attached to a research microscope (Leica Laborlux S Microscope, Wetzlar, Germany) at 200x magnification (field covered $21,670.9 \mu \mathrm{m}^{2}$ ). A total of $15-20$ fields from hippocampal regions (CA1-3 and CA4) and 30-40 fields from the subpial zone and the cortical ribbon (layers II-VI) from the frontal cortex were captured. These two zones were selected because of their proximity to the inflammatory exudates in the meningitic process and extension to the grey matter. All the labeled cell bodies of astroglial and microglial cells with distinct nuclei were counted manually in consecutive fields. The cell counts from all the frames were averaged and expressed as cell numbers of either type per $\mathrm{mm}^{2}$.

Area fraction of the immunolabeled zone in the sections stained for GFAP (astrocytic) and Iba1 (microglial), indirectly reflecting the sum of cells labeled and their staining intensity, was measured by using Image Analysis Software, Image J (public domain Java-based software developed by the National Institute of Health). The color images were converted to grey scale (256 shades of grey) for measurement.

\section{Statistical analysis}

The data was expressed as mean \pm standard deviation (SD). Analysis was carried out using SPSS software and the Mann-Whitney U test. $\mathrm{P}<0.05$ was considered significant.

Qualitative assessment of astrocytic and microglial cell response in different infections and association with HIV

Qualitative alterations in astroglia and microglia were assessed and compared between various opportunistic infections with and without HIV in contrast to controls. Astrocytes were considered to be resting when they had small cell bodies and fine processes on GFAP immunolabeling, and reactive when they had enlarged cell bodies and thick processes accumulating GFAP. Dystrophic astroglia had beaded and fragmented processes in contrast to the reactive forms $[15,16]$.

Microglia were classified as resting (ramified), activated, amoeboid, and dystrophic in accordance with standard descriptions [17].

Table 2. Details of the antibodies used for immunohistochemistry

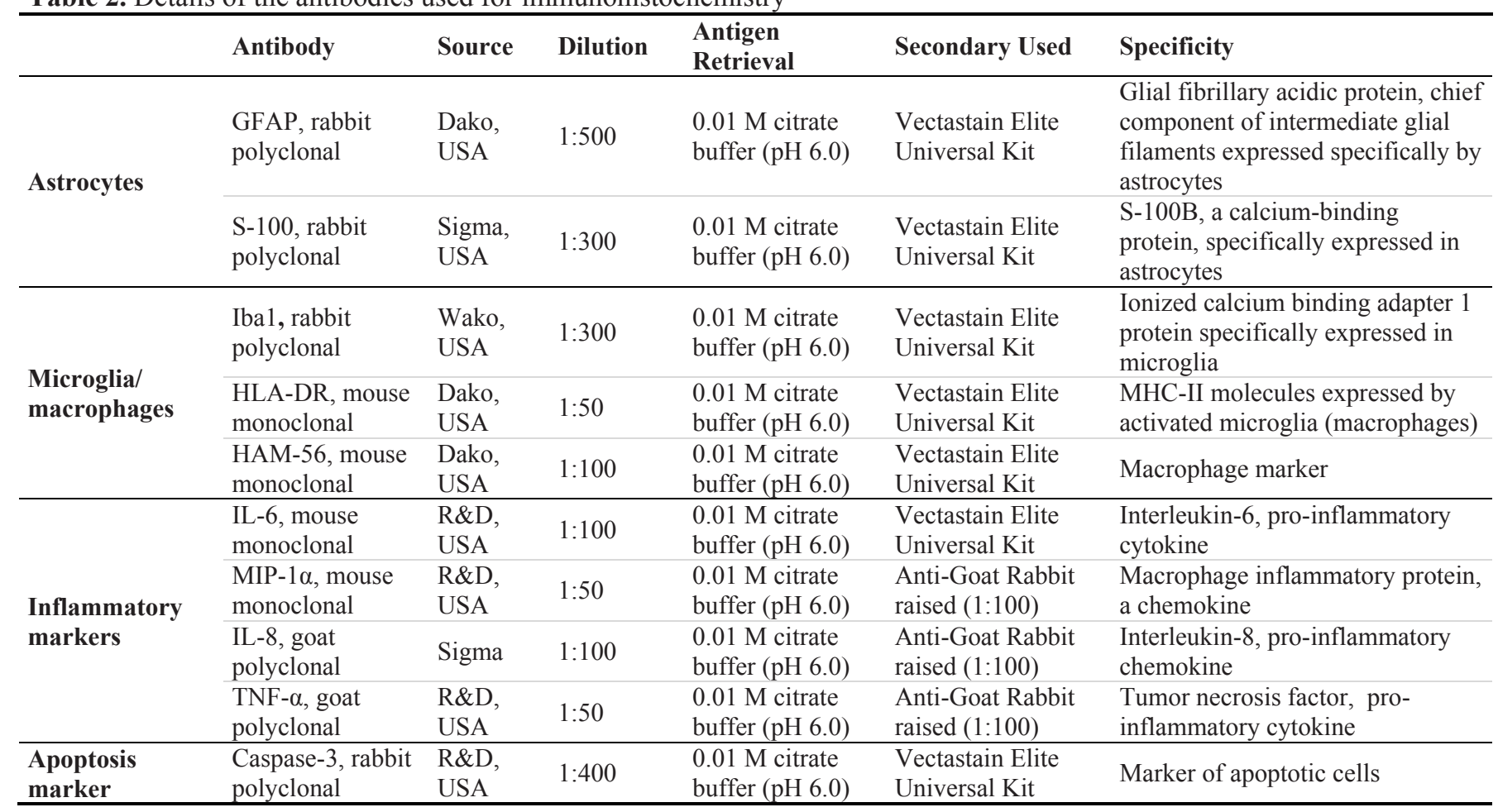




\section{Results}

Demographic and diagnostic features in various cases are represented in Table 1.

\section{Histopathological features}

Group $1(\mathrm{n}=5)$ consisted of normal controls. The brains selected from the controls derived from victims of head trauma were negative for HIV and other infections. They were essentially normal except for small focal contusions and subarachnoid hemorrhage.

Group $2(n=5)$ consisted of cases of HIV encephalitis without opportunistic infection. Bilateral diffuse frontal atrophy was seen in patients with HIV encephalitis (Figure 1A). No inflammatory exudates or opportunistic infections were detected on detailed histopathology. Two of the cases revealed features of HIV leucoencephalopathy, with demyelination in white matter, focal microglial nodules with multinucleate giant cells containing p24 antigen of HIV (Figure 5A), and mild perivascular mononuclear cell cuffing.

Group 3 consisted of cases of tuberculous meningitis, HIV positive $(n=5)$ and negative $(n=5)$. In HIV-negative TBM cases, basal inflammatory exudate of variable thickness was seen partially obscuring the cerebral vessels and cranial nerves (Figure 1B). The exudates were composed of aggregates of epitheloid cells and lympho-plasmacytic infiltrate in the subarachnoid space, focally breaching the pia-glial membrane and extending into a superficial molecular layer bordered by reactive astroglial cells (Figure 3A). In the hippocampus, the thick inflammatory exudates were noted entrapping branches of middle cerebral artery with granulomatous vasculitis and focal ischemic lesions.
In contrast, in cases of TBM associated with HIV (HIV-positive TBM), the basal exudates were thin (Figure 1C), but numerous acid-fast bacilli were seen in the exudates (Figure $1 \mathrm{C}$, inset). The inflammatory response lacked granulomas and was rich in histiocytes and lymphocytes (Figure 3F). A subpial carpet of reactive astrocytes had contained the subarachnoid inflammation, preventing the breach into the parenchyma. The perivascular inflammation was less compared to HIV-negative TBM cases. Focal zones of ischemic infarcts with demyelination were seen as a complication of arteritis.

Group 4 consisted of cases of cryptococcal meningitis, HIV positive $(n=5)$ and negative $(n=5)$. In HIV-negative cases of cryptococcal meningitis $(\mathrm{CM})$, a thick gelatinous mucoid exudate was seen covering the superolateral surfaces (Figure 1D). The exudates contained numerous budding yeast forms of Cryptococcus neoformans, expanding the subarachnoid and Virchow-Robin's space in the cortical ribbon. PAS and mucicarmine stains highlighted the polysaccharide mucinous capsule of the cryptococci (Figure 1D). The histiocytic response admixed with lymphocytes was brisk, with occasional multinucleated giant cells and histiocytes containing phagocytosed cryptococci (Figure 4D). The degrees of cellular infiltrate and fungi were more prominent near the hippocampal fissure. In cases of cryptococcal meningitis associated with HIV (HIV-positive CM), extracellular yeast forms were numerous, whereas lympho-histiocytic response was scarce and giant cell reaction was absent (Figure 4A). Ischemic and dystrophic changes in the neurons were conspicuously absent. Myelin pallor of the white matter was variable and mild compared to cases of TBM.

Table 3. Astroglial population $/ \mathrm{mm}^{2}$ in hippocampus and frontal cortex in various groups

\begin{tabular}{cccccc}
\hline \multirow{2}{*}{ Cases } & \multicolumn{2}{c}{ FRONTAL CORTEX } & \multicolumn{2}{c}{ HIPPOCAMPUS } \\
\cline { 2 - 6 } & Subpial region & Grey mater & CA4 & CA1-3 \\
\hline Controls & $0.420 \pm 0.0252$ & $0.192 \pm 0.0483$ & $0.461 \pm 0.036$ & $0.184 \pm 0.040$ \\
HIV without OI & $0.554 \pm 0.122$ & $0.391 \pm 0.15$ & $0.466 \pm 0.063$ & $0.161 \pm 0.023^{*}$ \\
TBM-HIV positive & $0.715 \pm 0.023^{*}$ & $0.338 \pm 0.070^{*}$ & $0.484 \pm 0.067$ & $0.323 \pm 0.056^{*} \$$ \\
TBM-HIV negative & $0.692 \pm 0.115^{*}$ & $0.323 \pm 0.138$ & $0.483 \pm 0.090$ & $0.252 \pm 0.060^{\$}$ \\
CM-HIV positive & $0.669 \pm 0.145^{*}$ & $0.280 \pm 0.180$ & $0.522 \pm 0.089$ & $0.258 \pm 0.071$ \\
CM-HIV negative & $0.553 \pm 0.070$ & $0.323 \pm 0.087^{*}$ & $0.531 \pm 0.071$ & $0.294 \pm 0.029 * \$$ \\
\hline
\end{tabular}


Graph 1. Graph denoting astroglial cell counts and area fraction in frontal cortex and hippocampus in different infectious conditions (with and without HIV infection), in frontal cortex subpial area (A), frontal cortex grey matter (B), hippocampus CA4 area (C), and hippocampus CA1-3 area (D).
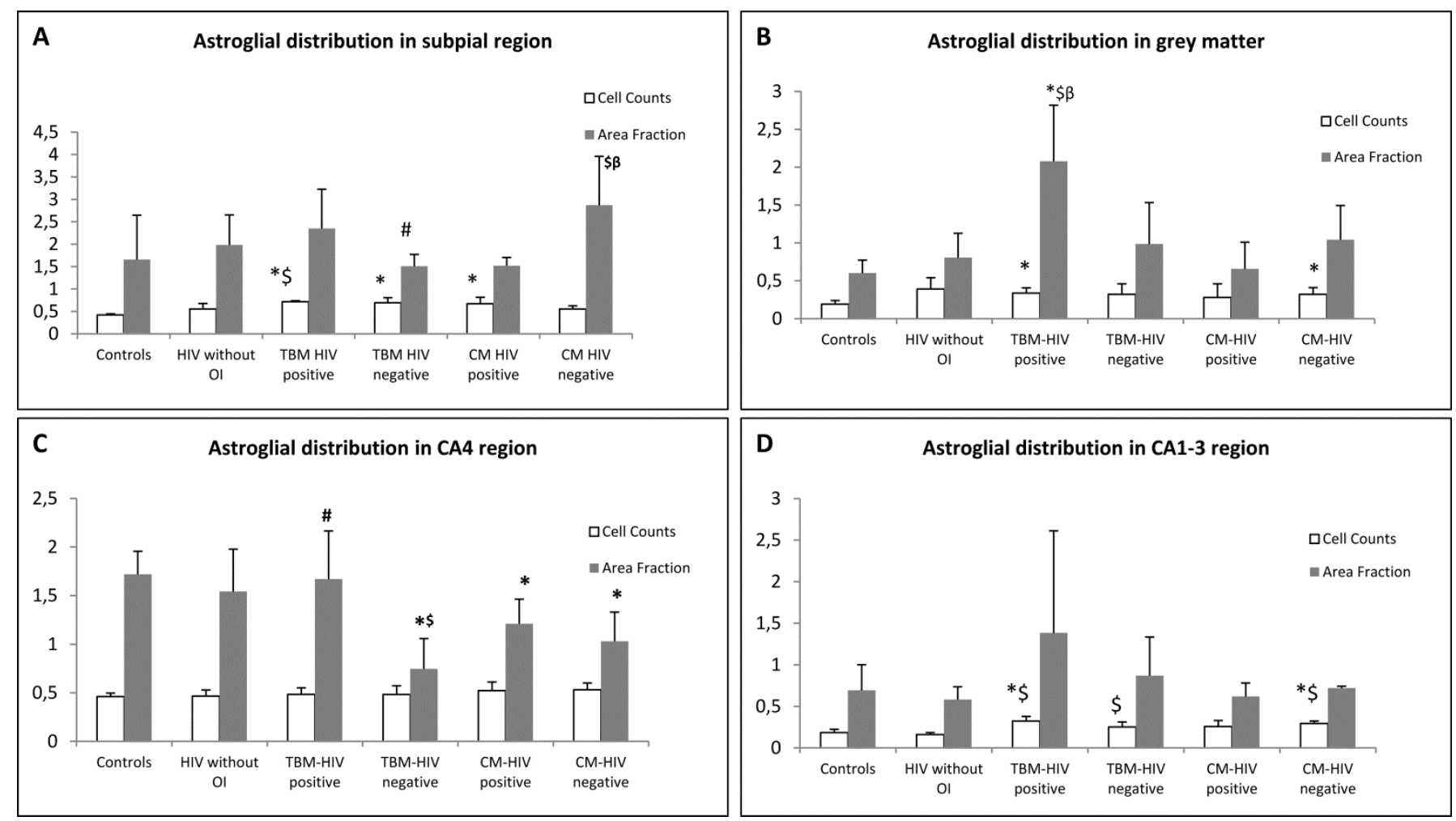

* = significance in comparison to controls; $\$=$ significance in comparison to HIV without OI; Significance tested by Mann Whitney $\mathrm{U}$ test, $\mathrm{p}<0.05 ; \mathrm{n}=$ $5 ; \mathrm{Y}$ axis $=$ cells $/ \mathrm{mm} 2$ for cell counts; percent immunostaining for area fraction.

Table 4. Area fraction of astroglia expressed as percent (\%) GFAP expression in hippocampus and frontal cortex (subpial region) in various groups

\begin{tabular}{ccccc}
\hline \multirow{2}{*}{ Cases } & \multicolumn{2}{c}{ FRONTAL CORTEX } & \multicolumn{2}{c}{ HIPPOCAMPUS } \\
\cline { 2 - 5 } & Subpial region & Grey mater & CA4 & CA1-3 \\
\hline Controls & $1.654 \pm 0.992$ & $0.601 \pm 0.172$ & $1.718 \pm 0.238$ & $0.692 \pm 0.310$ \\
HIV without OI & $1.981 \pm 0.674$ & $0.805 \pm 0.324$ & $1.542 \pm 0.435$ & $0.581 \pm 0.155$ \\
TBM-HIV positive & $2.347 \pm 0.879$ & $2.078 \pm 0.74 * \$ \beta$ & $1.669 \pm 0.496^{\#}$ & $1.384 \pm 1.227$ \\
TBM-HIV negative & $1.507 \pm 0.264^{\#}$ & $0.985 \pm 0.549$ & $0.745 \pm 0.314^{\$}$ & $0.868 \pm 0.467$ \\
CM-HIV positive & $1.518 \pm 0.183$ & $0.656 \pm 0.354$ & $1.209 \pm 0.254^{*}$ & $0.618 \pm 0.164$ \\
CM-HIV negative & $2.869 \pm 1.090^{\text {\$B }}$ & $1.042 \pm 0.452$ & $1.029 \pm 0.301 *$ & $0.719 \pm 0.024$ \\
\hline
\end{tabular}

Significance tested by Mann-Whitney $U$ test, $\mathrm{p}<0.05$; values are expressed as mean \pm SEM. $(*=$ significance in comparison to controls; $\$=$ significance in comparison to HIV without OI; \#=significance in comparison to the same with HIV infection; $\beta=$ in comparison to other infection (with/without HIV) 
Table 5. Microglial population $/ \mathrm{mm}^{2}$ in hippocampus and frontal cortex in various groups

\begin{tabular}{|c|c|c|c|c|}
\hline \multirow{2}{*}{ Cases } & \multicolumn{2}{|c|}{ FRONTAL CORTEX } & \multicolumn{2}{|c|}{ HIPPOCAMPUS } \\
\hline & Subpial region & Grey mater & CA4 & CA1-3 \\
\hline Controls & $0.369 \pm 0.095$ & $0.185 \pm 0.053$ & $0.298 \pm 0.12$ & $0.429 \pm .189$ \\
\hline HIV without OI & $0.300 \pm 0.186$ & $0.323 \pm 0.017^{*}$ & $0.427 \pm 0.096$ & $0.411 \pm .035$ \\
\hline TBM-HIV positive & $0.669 \pm 0.157^{*} \$$ & $0.507 \pm 0.064^{*} \mathrm{~s}$ & $0.692 \pm 0.175^{*} \mathrm{~s}$ & $0.453 \pm 0.105$ \\
\hline TBM-HIV negative & $0.601 \pm 0.068^{* \beta}$ & $0.458 \pm 0.096^{* \beta}$ & $0.555 \pm 0.125^{*}$ & $0.554 \pm 0.123$ \\
\hline CM-HIV positive & $0.382 \pm 0.039^{\$ \beta}$ & $0.290 \pm 0.071^{* \$ \beta}$ & $0.369 \pm 0.063^{\beta}$ & $0.321 \pm 0.174$ \\
\hline CM-HIV negative & $0.247 \pm 0.077^{\#}$ & $0.182 \pm 0.027^{\#}$ & $0.436 \pm 0.041$ & $0.463 \pm 0.033$ \\
\hline
\end{tabular}

Graph 2. Graph showing microglial cell counts and area fraction in different infectious conditions (with and without HIV infection) in frontal cortex and hippocampus regions in frontal cortex subpial area (A), frontal cortex grey matter (B), hippocampus CA4 area (C), and hippocampus CA1-3 area (D).
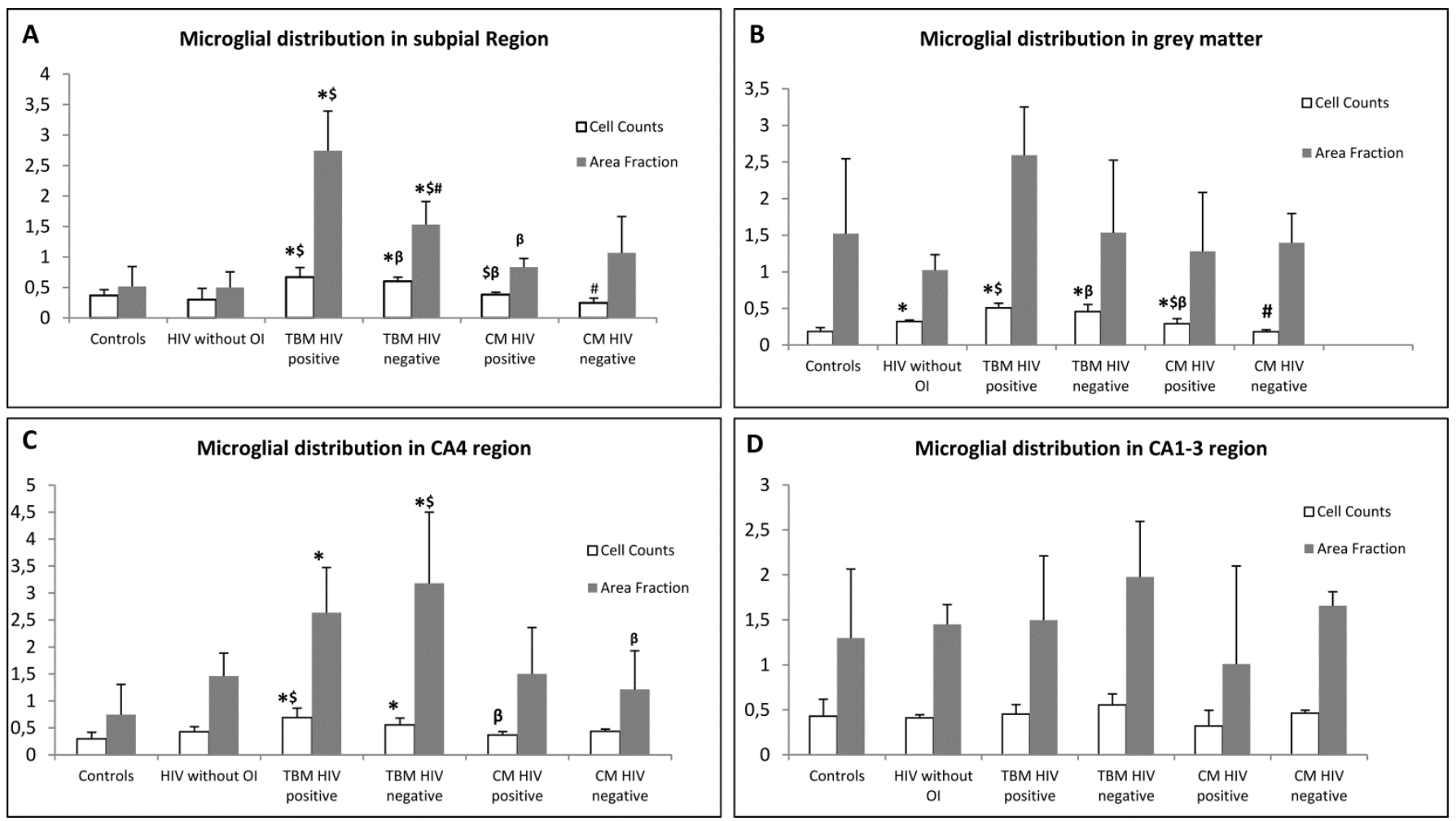

$*$ = significance in comparison to controls; $\$=$ significance in comparison to HIV without OI; \# = significance in comparison to same infection with/without HIV; B = in comparison to other infection (with or without HIV infection); Significance tested by Mann Whitney $\mathrm{U}$ test, $\mathrm{p}<0.05 ; \mathrm{n}=5 ; \mathrm{Y}$ axis $=$ cells $/ \mathrm{mm} 2$ for cell counts; percent immunostaining for area fraction. 
Table 6. Area fraction of microglia expressed as percent (\%) Ibal expression in hippocampus and frontal cortex (subpial region) in various groups

\begin{tabular}{ccccc}
\hline \multirow{2}{*}{ Cases } & \multicolumn{2}{c}{ FRONTAL CORTEX } & \multicolumn{2}{c}{ HIPPOCAMPUS } \\
\cline { 2 - 5 } & Subpial region & Grey mater & CA4 & CA1-3 \\
Controls & $0.515 \pm 0.326$ & $1.522 \pm 1.023$ & $0.745 \pm 0.56$ & $1.298 \pm 0.766$ \\
HIV without OI & $0.500 \pm 0.256$ & $1.023 \pm 0.211$ & $1.459 \pm 0.426$ & $1.450 \pm 0.219$ \\
TBM-HIV positive & $2.743 \pm 0.65^{*}$ s & $2.592 \pm 0.659$ & $2.633 \pm 0.84^{*}$ & $1.495 \pm 0.716$ \\
TBM-HIV negative & $1.531 \pm 0.378^{*} \$$ & $1.535 \pm 0.991$ & $3.18 \pm 1.32^{\$}$ & $1.976 \pm 0.618$ \\
CM-HIV positive & $0.832 \pm 0.145^{\beta}$ & $1.28 \pm 0.804$ & $1.50 \pm 0.86$ & $1.01 \pm 1.089$ \\
CM-HIV negative & $1.066 \pm 0.598$ & $1.396 \pm 0.40$ & $1.213 \pm 0.717^{\boldsymbol{\beta}}$ & $1.656 \pm 0.156$ \\
\hline
\end{tabular}

Significance tested by Mann-Whitney U test, $\mathrm{p}<0.05$; values are expressed as mean \pm SEM. $\left({ }^{*}=\right.$ significance in comparison to controls; $\$=$ significance in comparison to HIV without OI; \#=significance in comparison to the same with HIV infection; $\beta=$ in comparison to other infection (with/without HIV)

Figure 1. Gross photographs of HIV and opportunistic infections. (A) Diffuse cortical atrophy (pronounced in frontal lobes) with narrow gyri and deep sulci in a case of HIV encephalitis without opportunistic infections. Covering meninges is thickened. (B, C) Tuberculous meningitis with basal exudates entrapping cerebral vessels and cranial nerves in prepontine and optochiasmatic cistern in a HIV-negative individual (B, arrow). The basal exudates are thin $(\mathbf{C}$, arrow) in HIV-positive TBM but numerous acid-fast bacilli seen in exudates (C, inset). (D) Thick glistening mucoid exudates covering the superolateral convexity in a case of cryptococcal meningitis (D). Numerous budding yeast forms of cryptococci were detectable within the exudates (D, inset). [C, inset: Ziehl-Neelsen stain x Obj.100; D. inset: periodic acid-Schiff stain x Obi.40
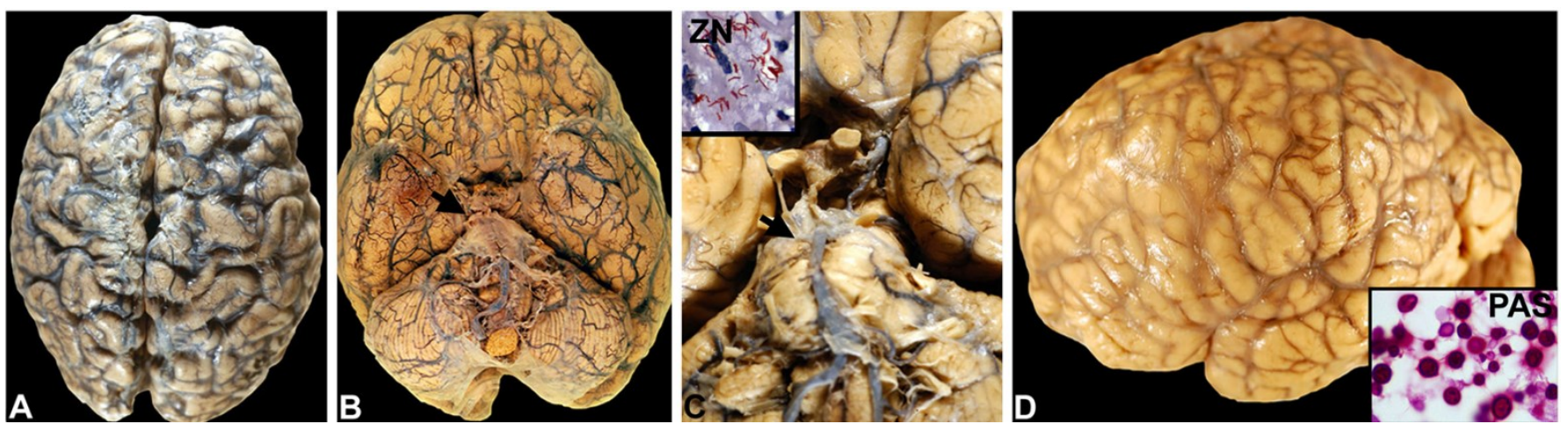

Figure 2. Astrocytic response in HIV encephalitis without OI. In comparison to control cases wherein the glial response was minimal (A), in cases of HIV encephalitis without OI, a thin band of subpial astrocytosis was seen (B). In the grey matter, reactive rounded hypertrophic forms were seen around microglial nodules (C). White matter revealed few astrocytes that were small and stunted, with dystrophic beaded processes (D). [Magnification: scale bar].

\section{HIV Encephalitis without OI}
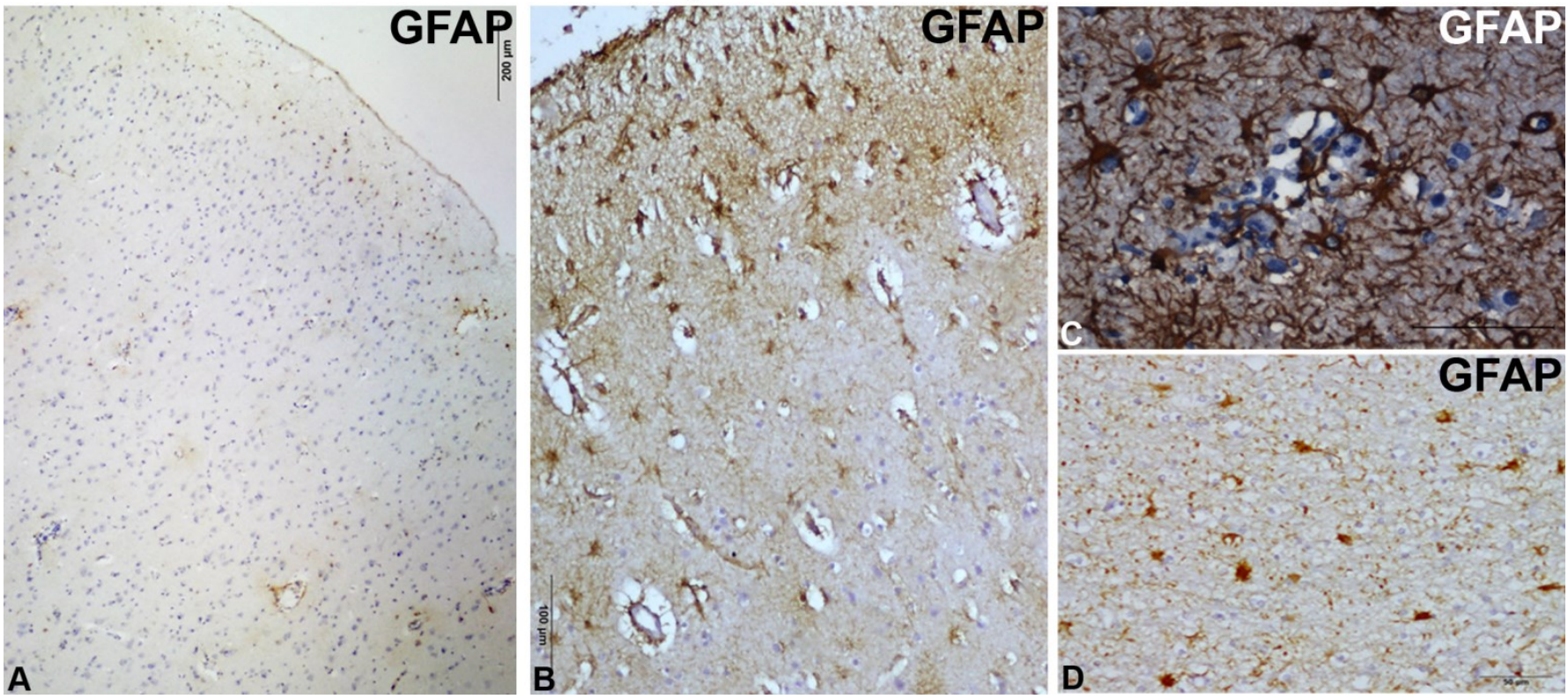
Figure 3. Histology and astrocytic response of TBM. HIV-negative TBM (A-E): Necrotizing granulomatous exudates and vasculitis seen in subarachnoid space (SAS) causing focal breach of pia-glial membrane and extension into superficial cortical layers (A). There is a band of reactive astrogliosis in the molecular layer beneath the exudate labeled by GFAP (B, *). S-100 $\beta$ in contrast shows diffuse labeling in cortex sparing the molecular layer (*) and perivascular zones, seen as pale unstained zones (C). Prominent astrocytosis seen around parenchymal epitheloid granulomas (D, E arrow), the astrocytes having rounded cell bodies and stunted processes (E, inset). HIV-positive TBM (F-J): The meningeal exudate lacked granulomas but had numerous acid-fast bacilli on Ziehl-Neelsen stain (F, inset). A thick band of reactive astrocytes seen in upper cortical layers I-III on GFAP (G), less prominent in S-100 $\beta$ and sparing the molecular layer $(\mathbf{H}, *)$. Astrocytes on GFAP stain had long slender processes (I) compared to small astrocytic cells with thin rims of cytoplasm and short processes on S-100ß (J). [Magnification: scale bar].
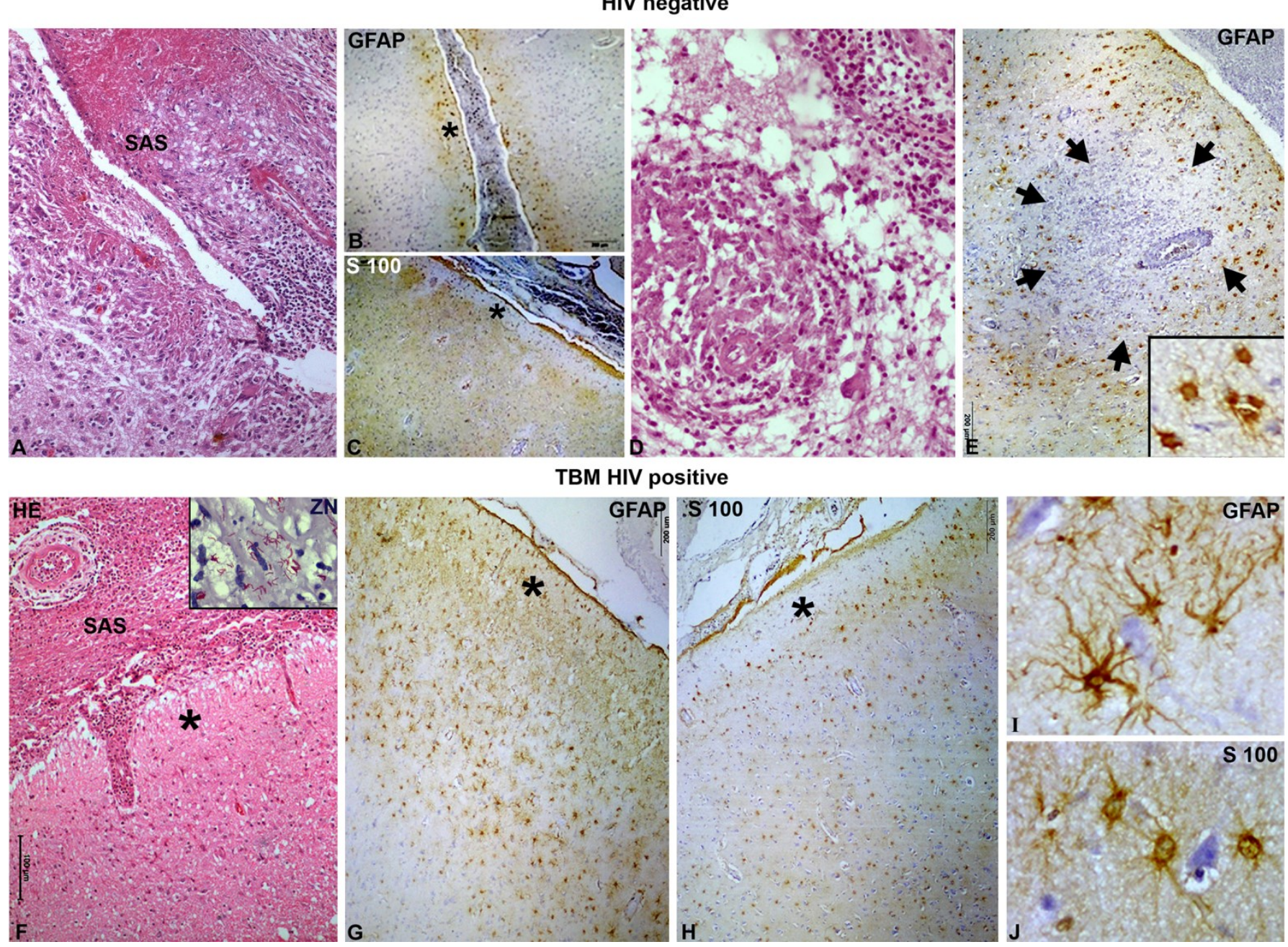
Figure 4. Histology and astrocytic response of crytpococcal meningitis (CM). HIV-positive CM (A-C): Cystic lesions with lakes of cryptococci seen in parenchyma with minimal inflammation and lacked giant cell response (A). A carpet of reactive astrocytes having large cell bodies and stunted processes expressing GFAP were found in subpial (B), perineuronal (B, inset) and perivascular in distribution. On S-100 $\beta$ immunolabeling, astrocytes in molecular layer were minimally labeled in contrast to layer II (C). HIV-negative CM (D-F): Meningeal exudate revealed cryptococcal yeast forms phagocytosed by macrophages and giant cells (D, arrow). GFAPlabeled reactive astrocytes aggregated around cryptococcal parenchymal cysts along the margin (E). Several astrocytes had irregular thick beaded processes and granular fragmentation resembling astrocytic plaques in grey and white matter (F). [Magnification: scale bar].
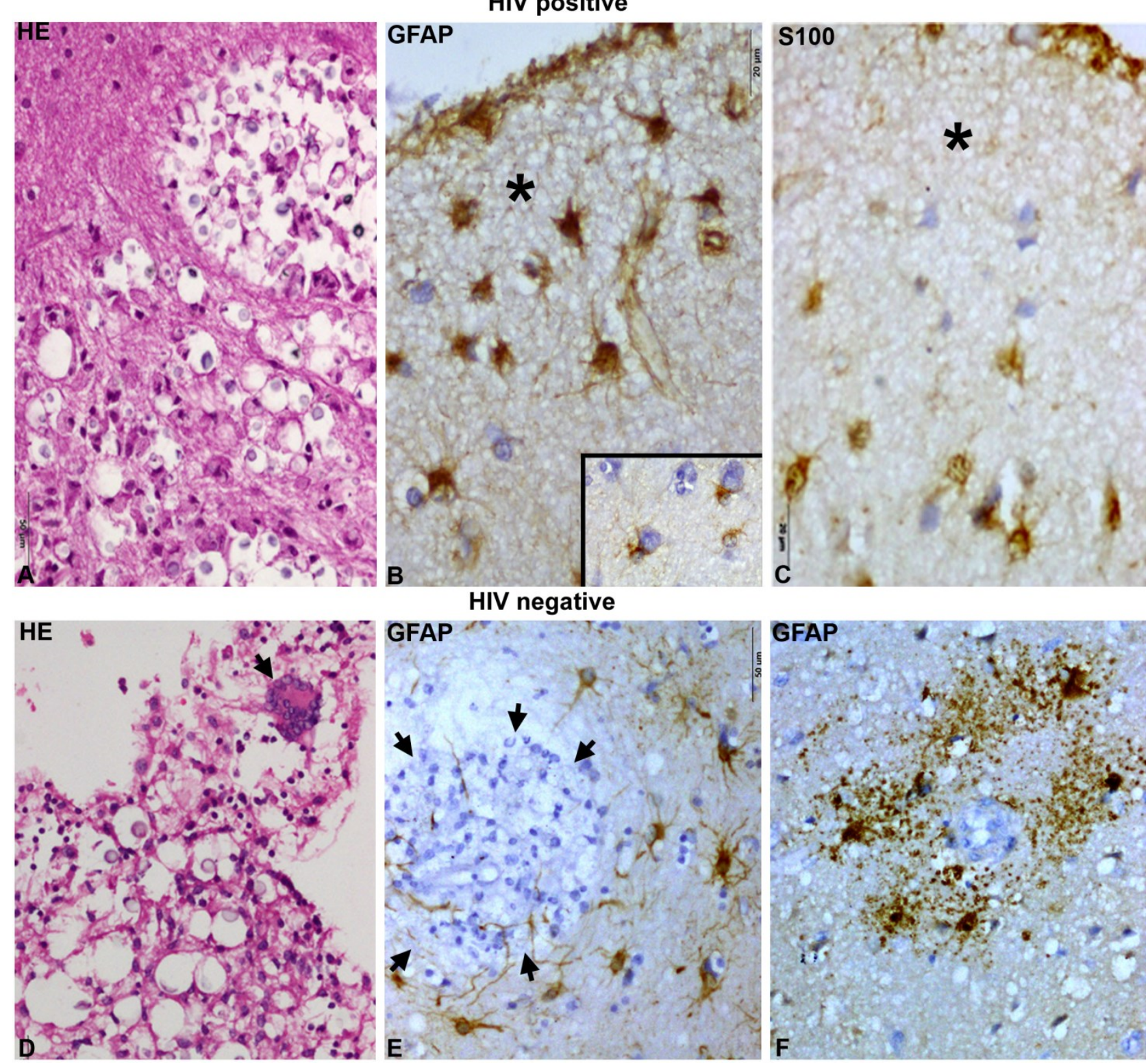
Figure 5. Microglial response in cases of HIV encephalitis without OI. Microglial nodules in the grey matter (A) containing p24 antigen (A, inset) were strongly labeled by Iba1 (B) and HLA-DR (C), indicating immune activation of microglia. Diffuse Ibal labeled microglia in grey matter in perineuronal distribution (B, inset) [Magnification: scale bar].
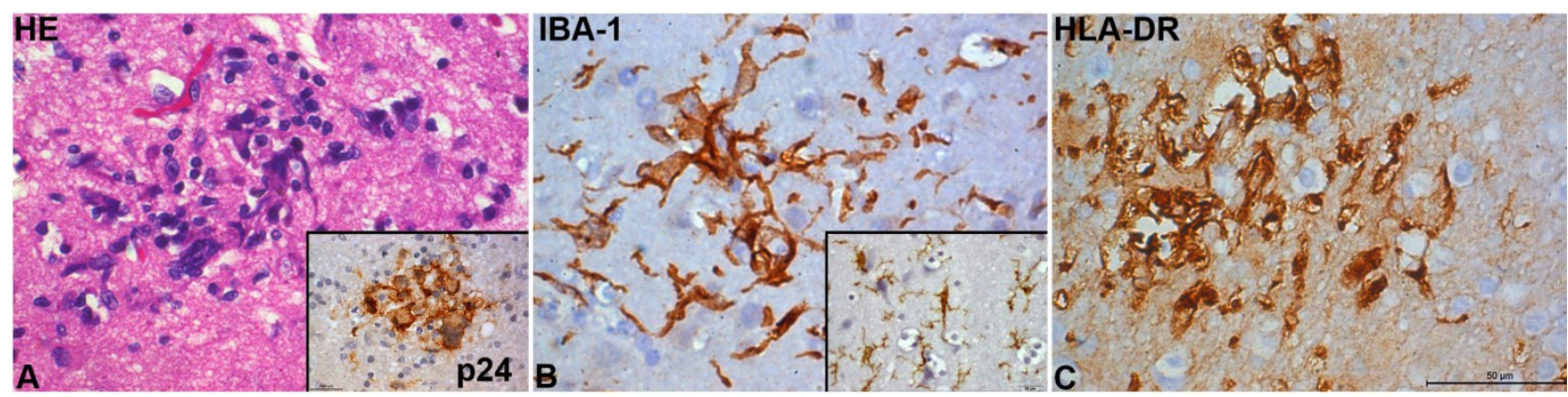

Figure 6. Meningeal exudate and microglial response in TBM. HIV-positive TBM (A-H): Diffuse lymphomononuclear exudates in the meninges were seen (A), the macrophages labeled with Iba-1 (B), HLA-DR (C), and HAM-56 (D). In the parenchyma, florid microglial response was seen in the cortex (F) beneath the exudate, breaching the pia-glial membrane (E) with activated phenotype and ramified processes (F). In addition, microglial nodules in the cerebral cortex were labeled with Iba-1 (G) and HLA-DR (H). HIV-negative TBM (I-L): Parenchymal granuloma in cerebral cortex (I) has Iba-1 labeled microglia with amoeboid morphology reflecting chronicity (J). Activated microglia are seen unsheathing neurons $(\mathrm{K}, \mathrm{L})$. The microglia close to granulomas show stunted processes surrounding ischemic neurons $(\mathbf{K})$ or display ramified processes enclosing neurons away from granulomas (L) [Magnification: scale bar].
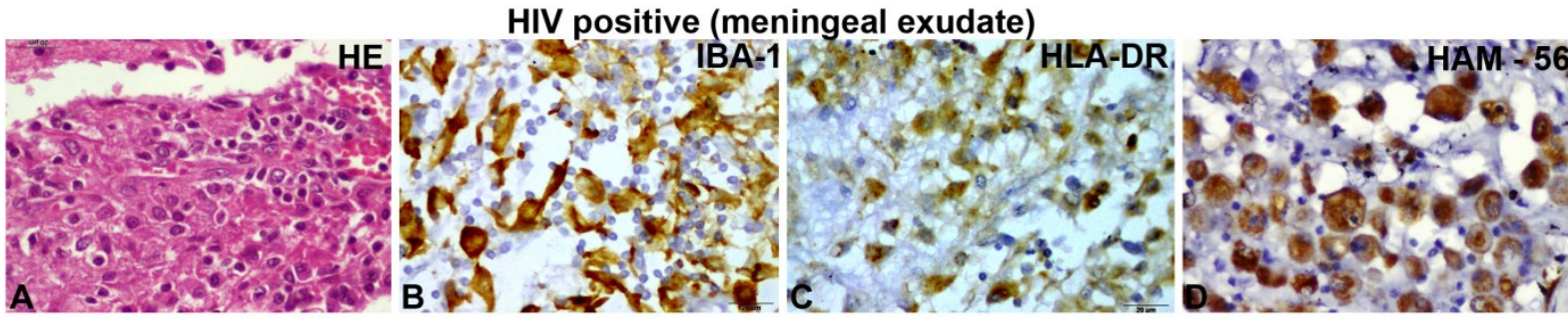

HIV positive (parenchymal alterations)
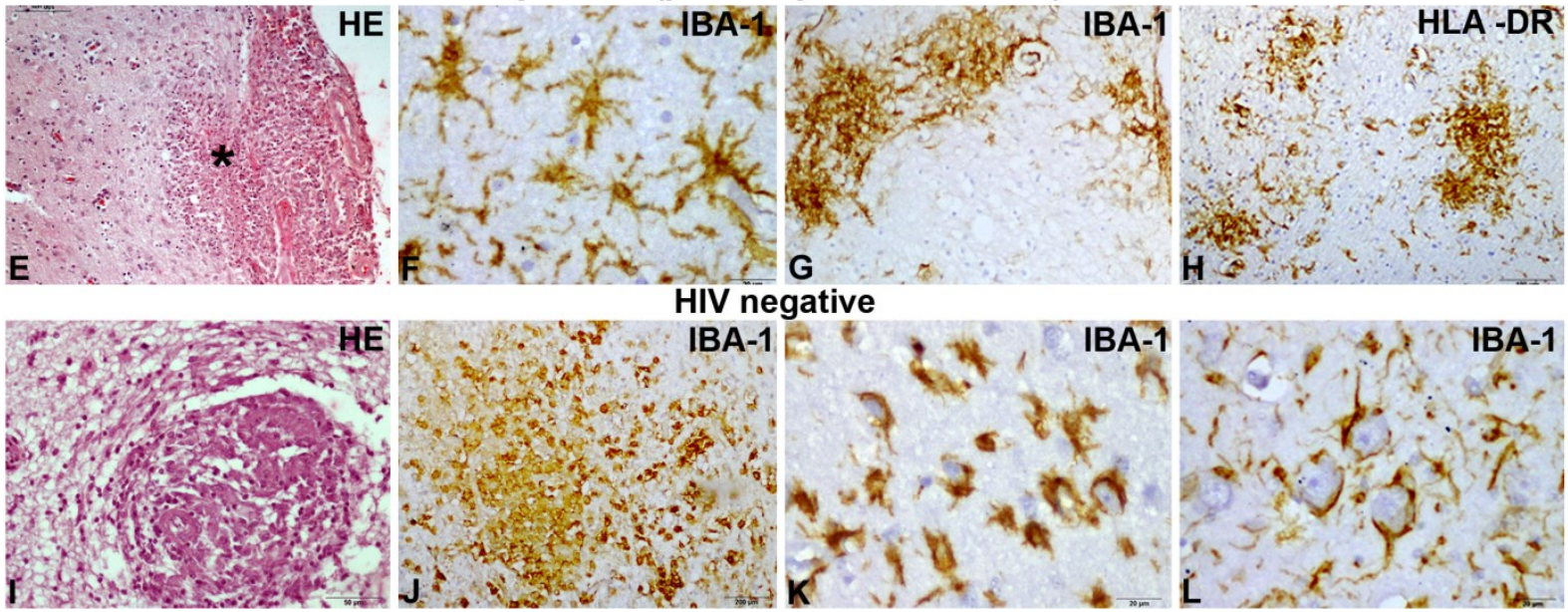
Figure 7. Microglial response and meningeal exudate in cryptococcal meningitis. HIV-positive CM (A-C): Iba-1labeled macrophage response is seen around the parenchymal pseudocysts (A) that are activated, HLA-DR positive (B), and admixed with hematogenously derived macrophages that are HAM-56 positive (C). HIV-negative CM (D-F): The meningeal exudate and giant cells (arrows) are labeled with antibody to Iba-1 (F) and HLA-DR (G). Some of the macrophages are hematogenous in origin and labeled by HAM-56 (H) [Magnification: scale bar].

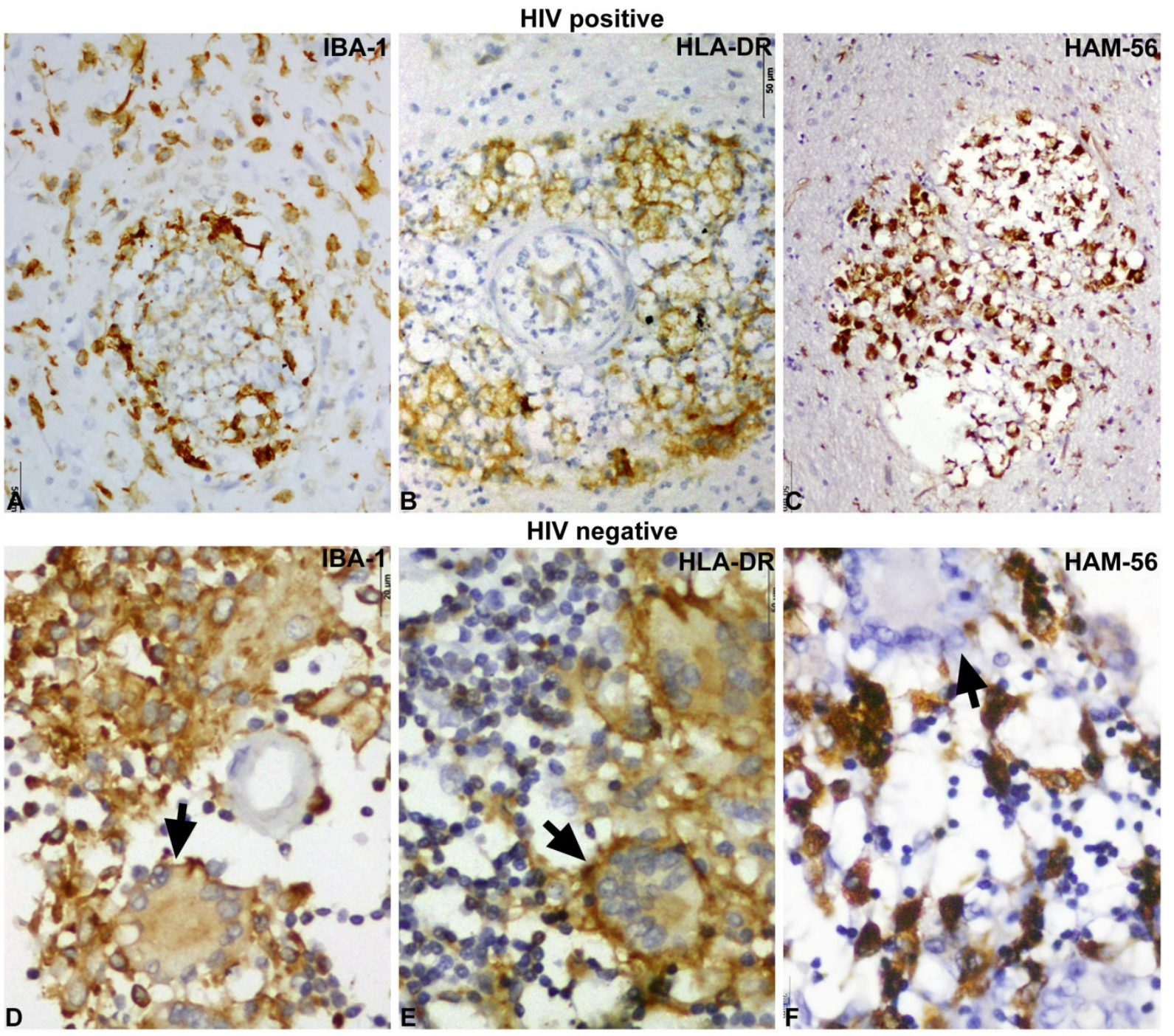


Figure 8. Caspase-3 immunolabeling in controls, HIV encephalitis without OI, TBM (HIV positive and negative) and CM (HIV positive and negative) cases. HIV without OI (B,C): Caspase-3 immunolabeling was negative in control except for occasional glial nuclei (A), while in cases of HIV encephalitis without OI, neuronal nuclear positivity in hippocampus (B) and oligodendroglia in white matter were noted (C). TBM (D-G): In cases of TBM, caspase-3 immunolabeling was detected in subpial astrocytes (D), occasional pyramidal neurons of temporal cortex (E), microglial cell processes ensheathing the neurons (F), and oligodendroglia nuclei in white matter (G). Cryptococcal meningitis (HJ): Caspase-3 positivity was detected in astrocytes bordering the cryptococcal pseudocyst $\mathbf{( H )}$. In cases of HIV-positive $\mathrm{CM}$, nucleus and cytoplasmic processes of microglia (I, inset) forming nodules in dentate gyrus of hippocampus were caspase-3 positive (I) Pyramidal neurons revealed nuclear labeling (J) in cases of both CM-HIV positive and negative [Magnification: scale bar; E, F, G: Obj x 40; I, inset:Obj. x 40].

\section{Control}

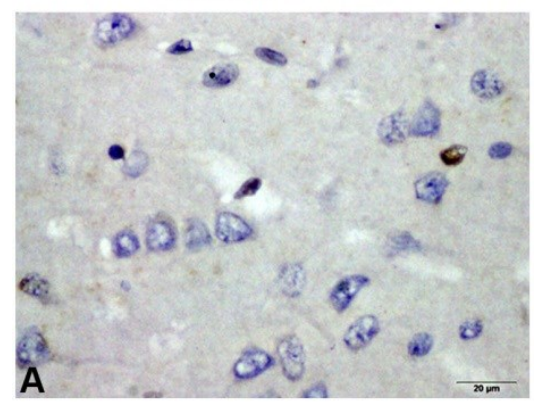

A

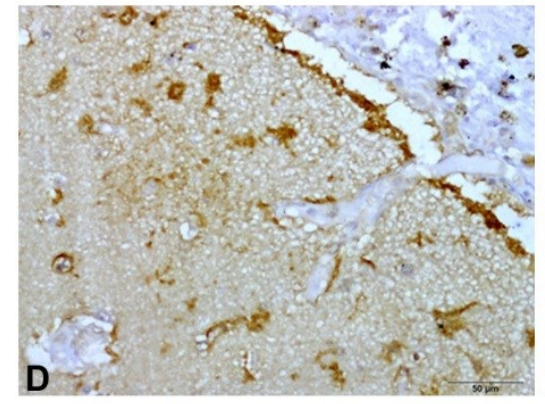

\section{Caspase-3 immnolabelling}

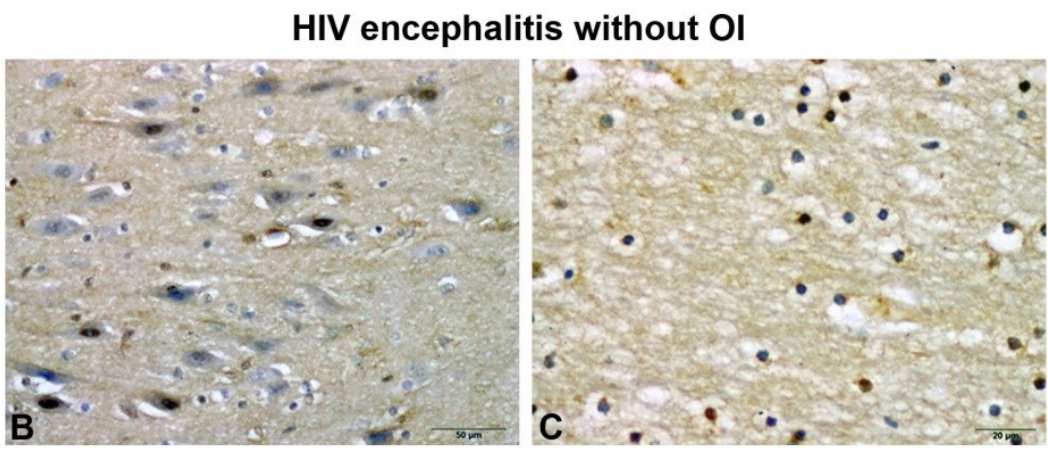

\section{Tuberculous meningitis (TBM)}
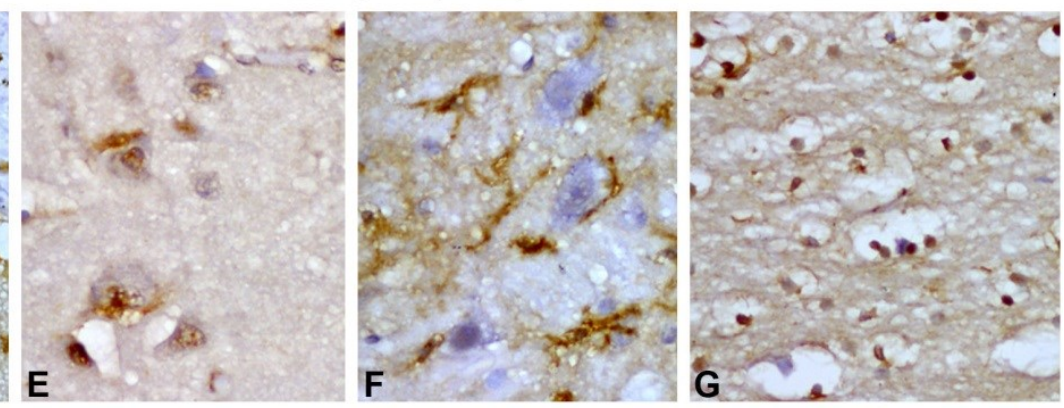

\section{Cryptococcal meningitis (CM)}
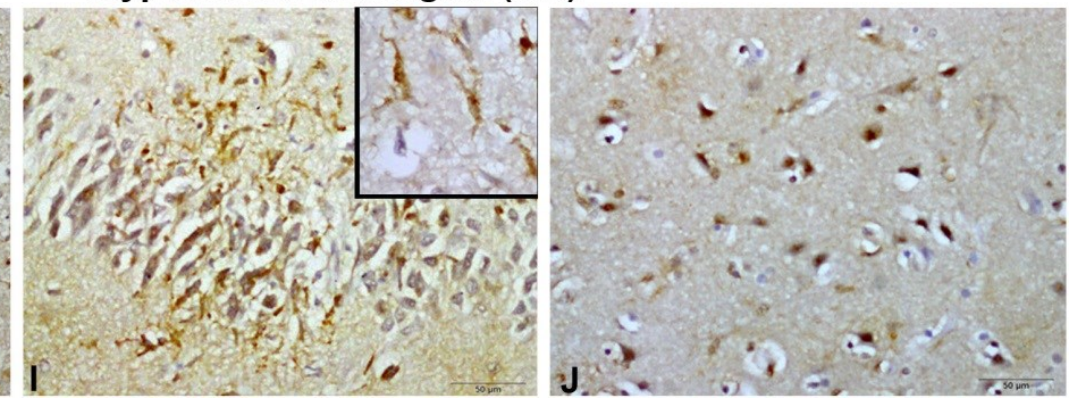


\section{Astroglial alterations}

In the frontal cortex of normal control samples, GFAP immunolabeled subpial astrocytes and the piaglial membrane (Figure 2A). In the underlying cortical ribbon and white matter, astrocyte cells were fewer in number and mostly confined to the perivascular zones (Table 3). In the grey matter, there was a predominance of protoplasmic astrocytes compared to fibrous astrocytes with smaller cell bodies and slender long branching processes in the white matter. In the hippocampus, fibrous astrocytes were seen in the hilus of Ammon's horn, extending their processes through the dentate gyrus into the overlying molecular layer. The astrocytes were seen diffusely distributed, ensheathing blood vessels.

In control cases, S-100 $\beta$, another astrocytic marker (calcium-binding protein in astroglial cytoplasm) highlighted glial cells with small cell bodies and sparse, thin and short processes. The intensity and distribution pattern differed from GFAP. In perivascular zones, while GFAP labeled astrocytic foot processes impinging on the vessel wall, S-100 $\beta$ staining revealed perivascular unstained cuffs with sparse or absent cell processes. Similarly, the diffusely distributed astrocytes in the cortex and subpial zone highlighted by GFAP were contrasted by relatively less staining with S-100ß. The grey-white junction was conspicuous as a broad labeled band, indicating junctional gliosis. Fibrous astrocytes with long thin processes were observed in the white matter.

In cases of HIV encephalitis not associated with opportunistic infections, the astrocytic distribution in the subpial zone was mildly increased compared to normal uninfected controls (Figure 2B). Morphologically, the astrocytes were reactive with enhanced GFAP labeling of the branching cytoplasmic processes, covering a wider surface area (higher area fraction) than the controls (Figure 2C) (Table 4; Graph $1 \mathrm{~B}$, not statistically significant). In the grey matter, astrocytosis was both diffuse and perivascular in distribution, with thick glial processes forming a limiting membrane around the vessels, suggesting a response to an altered blood-brain barrier. Astrocytes surrounding the microglial nodules in the white matter had an activated phenotype with small rounded gemistocytic forms and short but bushy processes, while astrocytes in the white matter displayed fragmentation and beading of the branching processes, reflecting a dystrophic change (Figure 2D). In the hippocampus, the astrocyte count in the Ammon's horn (CA1-3) region was significantly higher compared to normal controls $(\mathrm{p}<0.05$ ) (Table 3;
Graph 1D), although there was no significant increase in the area fraction (Table 4; Graph 1D). No statistically significant increase in astrocyte numbers was noted in the CA4 region or in the hilum of the dentate gyrus compared to controls.

S-100 $\beta$ labeling was similar to, but less intense than GFAP in this group (figure not shown). Akin to GFAP labeling, S-100 $\beta$ also highlighted the dystrophic astrocytic processes in the white matter as fragmented and tortuous forms. S-100 $\beta$ did not, however, label reactive astrocytes encircling microglial nodules, reflecting differential functional expression.

In cases of TBM, prominent subpial astrocytosis was evident beneath the meningeal exudates (Figures $3 \mathrm{~A}, \mathrm{~F})$ in cases with or without HIV compared to the controls; the increase was higher in the HIV-positive TBM group $(\mathrm{p}<0.05)$ (Figure 3G; Table 3; Graph 1A). The astrocytes aggregated beneath the subarachnoid inflammatory exudate, breaching the pia-glial membrane and the upper cortical layers, attempting to wall off the inflammatory response with a decreasing gradient of astrocytosis towards the lower layers (Figures 3B,G).

In the HIV-negative TBM cases, the astrocytes surrounded the parenchymal granulomas (Figure 3D) and had small rounded hypertrophied cell bodies with intense GFAP labeling and short stout processes (Figure 3E), resulting in a lower area fraction. Perivascular astrocytosis in grey and white matter demonstrated more numerous and thicker branching processes. In the HIV-positive cases, the astrocytic processes were long, slender, and more conspicuous, resulting in higher area fraction $(\mathrm{p}<0.05)$ (Figure 3I; Table 4; Graph 1B).

In the hippocampus, higher numbers of astrocytes were seen in the CA1-3 subfields in both HIVpositive and HIV-negative TBM groups compared to the controls and cases of HIV without OI $(\mathrm{p}<0.05)$ (Table 3; Graph 1D). The astrocytic morphology was essentially similar to respective frontal cortices. The area fraction of astrocytes in the CA4 zone of HIVpositive TBM cases was significantly higher than that of HIV-negative TBM cases $(\mathrm{p}<0.05)$ (Table 4; Graph 1C). The astrocytic processes in white matter revealed dystrophic changes (more so in the frontal cortex than the hippocampus), though this was less evident in HIV-positive TBM cases. Perineuronal presence of astrocytes was noted in both the groups; the processes were invaginated in between the neurons.

In HIV-negative TBM cases, in the grey matter, the subpial and perivascular zones had unstained pale 
cuffs compared to dense diffuse neuropil labeling (Figure 3C). Along the grey-white junction, a dense, diffuse band of S-100 $\beta$-labeled neuropil zones was noted. The density of labeling by S-100 $\beta$ was distincltly lower than GFAP and the labeled cells had small cell bodies with thin rims of cytoplasm and fine processes.

In HIV-positive TBM cases, S-100 $\beta$ stained the neuropil in the grey and white matter without astrocytosis, with unstained subpial zones below the inflammatory exudate (Figure $3 \mathrm{H}$ ). Morphologically, the labeled astrocytes had small round cells with stunted processes; the cell density was variable, but at more places than GFAP-labeled cells (Figure 3J).

In cases of HIV-positive cryptococcal meningitis, the subpial zone beneath the meningeal exudates with budding yeasts showed a significantly higher degree of astrocytosis compared to normal controls $(\mathrm{p}<0.05)$ (Figures 4A,B; Table 3; Graph 1A). Perineuronal and perivascular astrocytosis was more prominent in HIVpositive $\mathrm{CM}$ cases (Figure 4B). No significant difference was found in the astrocytic cell counts or area fraction by GFAP immunolabeling, between HIV-positive and HIV-negative CM cases, either in the frontal cortex or the hippocampus (Tables 3,4). However, diffuse and perivascular astrocytosis in both HIV-positive and HIV-negative cases in grey matter was higher compared to that in controls $(\mathrm{p}<0.05)$.

Morphologically, in contrast to cases of TBM, activated hypertrophic astrocytes with rounded cell bodies and retracted processes were seen in HIVpositive CM cases (Figure 4B), whereas in HIVnegative $\mathrm{CM}$ cases, astrocytes had prominent branching processes. In HIV-negative CM cases, gliosis and reactive astrocytosis were noted to be encircling the cystic cryptococcal parenchymal lesions (Figure 4E), and this feature was conspicuously minimal in HIV-positive CM cases. In addition, focal zones showed irregular breakdown and beading of astrocytic processes forming plaque-like zones, suggesting dystrophic change of glial cells in superficial and mid zones of the frontal cortex similar to cases of TBM (Figure 4F). In the hippocampus, the astroglial cell counts in Ammon's horn (CA1-3 zone) were higher in the HIV-negative group $(\mathrm{p}<0.05)$ compared to HIV-OI and control cases but essentially close to HIV-positive CM cases (Table 3; Graph 1C,D).

The pattern of expression in cryptococcal meningitis was similar in both HIV-positive and HIVnegative cases. The pia-glial membrane was strongly labeled by S-100 $\beta$ similar to GFAP, indicating surface gliosis, while the molecular layer of the frontal cortex had a conspicuous pale band unlabeled by S-100 $\beta$ (Figure 4C). There was no significant astrocytosis around parenchymal lesions but for a thin rim of diffusely labeled zone for S-100 $\beta$. The density of S$100 \beta$-positive astrocytes was low, though diffuse. The subcortical white matter was labeled as a dense S$100 \beta$-positive diffuse band with minimal cellular elements. While the deep white matter had variable S$100 \beta$-labeled astrocytosis with greater concentration around the vessels, it was less intense than in TBM cases.

\section{Microglial changes}

In the control cerebral cortex and hippocampus, diffuse distribution of resting/ramified microglia with very fine, thin branching processes and small cell bodies, labeled by Iba1, were seen throughout the grey and white matter. No perivascular or subpial aggregates were noted. Rare HLA-DR-labeled activated microglia were noted along the vessel walls in the grey matter of the frontal cortex, but none were found in the subpial zone or deep parenchyma. No HAM-56 labeled (marker for systemic circulation derived macrophages) cells were found.

In the frontal cortex from cases of HIV encephalitis with no associated opportunistic infections, a diffuse increase in microglia were found in the grey matter compared to normal controls $(p<$ 0.05) (Table 5; Graph 2B) with reactive ramified morphology, at places forming microglial nodules (Figures 5A,B) close to blood vessels and enclosing neurons. The cells forming microglial nodules in the white matter were labeled by Iba1, HLA-DR, and p24 (Figures $5 \mathrm{~A}-\mathrm{C}$ ) but rarely by HAM-56, indicating their origin from native brain-derived microglia, of which some were infected by HIV (Figure 5A, inset). The white matter had a lower density of microglia, with resting ramified forms resembling the ones in a normal cortex.

In the hippocampus, an increase in reactive ramified microglia was seen throughout CA1-4 zones of Ammon's horn, and was also seen infiltrating the dentate gyrus. No statistically significant increase in area fraction was noted either in the frontal cortex or hippocampus on Iba1 labeling for microglia (Table 6; Graphs 2A-D). Activated HLA-DR-positive microglial cells were found in the perivascular and subpial zones, abutting the pia-glial membrane. However, no HAM56-labeled macrophages were noted in the above representative areas, reflecting the absence of systemic macrophages in the pathological process. 
The macrophages in the meningeal exudates in cases of HIV-positive and HIV-negative TBM had strong Iba1, HLA-DR, and HAM-56 labeling (Figures 6A-D). The pia-glial membrane was invested by a layer of HLA-DR-positive cell processes, reflecting activation and an attempt to contain the spread of inflammatory signals. In cases of HIV-positive and HIV-negative TBM, numerous microglia were seen beneath the meningeal exudates and in the grey matter with a decreasing density towards the deeper cortex when compared to the controls. In the subpial zone, the density of reactive ramified microglial number with thick processes was higher $(\mathrm{p}<0.05)$ (Table 5; Graphs 2A-D) and covered a wider surface area in the subpial region $(\mathrm{p}<0.05)$ (Table 6; Graph 2A).

In HIV-positive TBM cases, several microglial nodules with intense Iba1 and HLA-DR labeling were seen in the grey matter (Figures 6F-H) and fewer were seen in the white matter. In the white matter of the fronto-temporal cortex and hippocampus, beyond the subcortical U-fibers, several reactive and amoeboid forms were observed, suggesting the spread of activating soluble signals to microglia along the interstitial space. Though the microglial nodular aggregates close to the surface had Iba1, HLA-DR, and HAM-56 labeled cells within the brain parenchyma and perivascular space, HAM-56 positive cells were rare, indicating restricted participation of the systemic macrophage system in inflammatory response in the brain parenchyma in cases of tuberculous meningitis.

In HIV-negative TBM cases with well-formed epitheloid cell granulomas in the meninges (Figure 6I), diffuse microglial response was seen in the entire thickness of the grey mater. HAM-56 stained histiocyte cells were seen in the meningeal exudates and infiltrating the choroid plexus of the temporal horn. The microglia had an amoeboid appearance with retracted processes and rounded cell bodies surrounding parenchymal granulomas (Figures 6J,K), while dystrophic microglia were seen in the hippocampus. Increase in area fraction of Iba1-labeled microglia noted in both HIV-positive and HIVnegative TBM groups was statistically significant in the CA4 zone $(\mathrm{p}<0.05)$ in comparison to the controls and HIV-OI cases (Table 6; Graph 2C). The microglial activation was not prominent in the white matter, though some of the capillaries had rare HLA-DR positive cells along the walls. Perineuronal microglia were seen in both the groups (HIV-positive and HIVnegative TBM cases) (Figure 6L) with no distinct neuronophagia.
In cases of cryptococcal meningitis, the subpial zone and grey matter of the frontal cortex had lower numbers of microglial cells compared to TBM $(\mathrm{p}<$ 0.05) (Table 5; Graphs 2A,B).

In HIV-positive CM cases, the subpial area had a carpet of HLA-DR-positive microglia, while the perivascular cells were HLA-DR and HAM-56 positive, reflecting the admixture of brain-derived microglia and systemic macrophage system. Parenchymal cryptococcal lesions were surrounded and infiltrated by numerous Iba1-positive reactive amoeboid microglia (Figure 7A), some of which expressed HLA-DR (Figure 7B) and a few others that strongly expressed HAM-56 (Figure 7C). Randomly distributed microglial aggregates were seen in both the Ammon's horn subfields and white matter in HIVpositive CM cases. Morphologically, the microglia had a reactive ramified appearance, resembling those seen in HIV without OI. Amoeboid forms were restricted to perivascular and perineuronal satellites.

In HIV-negative CM cases, the macrophages and giant cells with ingested cryptococci in the subarachnoid space, choroid plexus and within the cryptococcal pseudocysts were strongly labeled with Iba1, HLA-DR, and HAM-56 antibodies (Figures 7DF). The Iba1-positive microglia in the subpial zone and around the pseudocysts in the HIV-negative group were admixture of resting, ramified, and occasional amoeboid forms, the majority of which expressed HLA-DR. The degree of HLA-DR labeling of the microglia on visual assessment appeared to be less than in HIV-negative TBM cases. The microglial cell counts in CA4 region of Ammon's horn in HIVpositive $\mathrm{CM}$ cases was significantly lower than that seen in HIV-positive TBM cases $(\mathrm{p}<0.05)$ (Table 5; Graph 2C). Lower area fraction in the CA4 subfield was noted in HIV-negative $\mathrm{CM}$ cases compared to HIV-negative TBM cases $(\mathrm{p}<0.05)$ (Table 6; Graph $4 C)$. In the presence of parenchymal cryptococcal lesion, activated microglia were seen both within and surrounding the lesion in the HIV-negative CM cases, whereas it was restricted to perilesional zones in the HIV-positive CM cases.

\section{Caspase-3 immunolabeling}

Caspase-3, a cellular apoptotic mediator, labeled occasional astrocytic nuclei in the grey and white matter in the control samples (Figure 8A). Neurons were unlabeled in all anatomical areas studied. In HIV-positive cases without associated opportunistic infections, caspase 3 labeling was rarely found in random neurons (Figure 8B) and occasional 
oligodendrocytes in white matter (Figure 8C), but it failed to label microglial nodules in cases of HIV encephalitis.

In HIV-positive TBM cases, caspase-3 labeled the astrocytic nuclei in the subpial zone (Figure 8D), occasional pyramidal neurons (Figure $8 \mathrm{E}$ ) of the Ammon's horn close to exudate, microglia, oligodendroglia and astroglia (marginally more in the grey than white matter), and occasional endothelial cells in the small vessels. In HIV-negative TBM cases, caspase-3 expression was noted in the astrocytes adjacent to the granulomas in the frontal cortex and hippocampus, but less so in parenchymal microglial cells. Curiously, perineuronal and perivascular glial cell processes were labeled for caspase- 3 in the hippocampus and temporal cortex (Figure 8F). The edematous and anoxic areas in the white matter had caspase-3-labeled oligodendroglial and astrocytic nuclei (Figure 8G).

In cases of cryptococcal meningitis with HIV, caspase-3 labeling was observed focally in subpial astrocyte nuclei, perivascular microglial cells, and occasional neurons. The reactive microglial nodules in the hippocampus and white matter had cytoplasmic labeling, while the oligodendroglia and astrocytic nuclei in white matter were stained, indicating apoptotic cell loss of glial elements.

In cryptococcal meningitis-HIV-negative cases, caspase-3 immunostaining was noted in subpial astrocytes, astrocytes bordering the early infarct, and fungal pseudocysts (Figure $8 \mathrm{H}$ ). One case with infarct revealed nuclear labeling of the neurons (Figure 8I), indicating ischemia-induced apoptotic neuronal death. In the temporal cortex, focal perineuronal and perivascular microglial cells were caspase-3 positive. White matter showed randomly distributed caspase-3 positivity in microglia in addition to oligodendroglia nuclei (Figure 8J).

\section{Discussion}

It is generally believed that the astrocytic and microglial responses to chronic infections of the central nervous system (CNS) are essentially similar [18]. However, information about the differences in morphological, phenotypic and protein expression profiles following opportunistic infections such as tuberculosis and cryptococcosis and the influence of HIV infection is lacking. The uniqueness of the present study is the elucidation of pathomorphological features of astroglia and microglia in cases of chronic meningitis caused by Mycobacterium tuberculosis and Cryptococcus neoformans, in association with HIV, in human brains collected postmortem. The features were compared with control brain tissue collected from victims of road traffic accidents without associated CNS infections and from subjects with HIV encephalitis without concurrent opportunistic infection. Both the glial elements are now recognized to take active part in immune mediation and modulation in the central nervous system, guiding the course of evolution of pathomorphological features.

Astrocytes in response to any pathological insult in the brain transform into reactive forms, leading to either hypertrophy, proliferation, and/or overexpression of GFAP $[19,20]$. In the present study, a similar phenomenon was observed following chronic meningitic process with diffuse astrocytosis and hypertrophy, maximal in the subpial zone beneath the meningeal exudates in both cases of tuberculous and cryptococcal meningitis. A decreasing gradient of glial cell density, GFAP and S- $100 \beta$ expression, was evident in the lower cortical layers, suggesting a spread of transducing signals from the inflammatory focus along the surface. In both tuberculous and cryptococcal meningitis, dense glia limitans with gliosis was noted, indicating the efforts to restrict the spread of inflammatory process into the adjacent parenchyma by the pia-glial limitans. Breach of the glia limitans can be induced by hydrolyzing and proteolytic substances released by inflammatory infiltrate, especially by polymorphonuclear leukocytes and histiocytes [21] acting on surface matrix proteins.

$\mathrm{S}-100 \beta$ is an astrocyte-specific calcium-binding protein, the upregulation of which may lead to neuronal damage [22-24]. Several earlier studies have established that S-100 $\beta$ exerts a neuroprotective and neurotrophic influence at nanomolar concentrations [25-27], but at micromolar concentrations it is toxic, leading to astrocytic and neuronal death $[22,28]$. Esposito et al. [29] observed that S-100 $\beta$ protein initiates a gliotic response by inducing the release of pro-inflammatory mediators such as nitric oxide (NO) and cytokines from the microglia and astrocytes, which sequentially are deleterious to neurons. It is likely that S-100 $\beta$-labeled reactive astrocytes and the diffuse neuropil labeling noted in the cortical grey matter and hippocampus in cases of TBM and CM may have a slowly evolving toxic effect with apoptosis on neurons, though not overtly evident.

In HIV-positive CM cases, the host inflammation is minimal, unlike in mycobacterial infection cases [30]. Berger et al. [31] reported that increase in the astrocytic proliferation or activation around cryptococcal cystic lesions in the brain was not 
evident, as noted in the adjacent unaffected parenchyma. In the present study, a differential reactive astrocytic response was observed around the parenchymal cryptococcal pseudocysts, the reactive change being less when associated with HIV. The immuno-compromised state of HIV probably influences the cytokine-induced astrocytic proliferation in cases of cryptococcal infection.

Microglia are recognized as the main inflammatory cells of the CNS and play a crucial role in responding to CNS damage [32-34]. Our results with Iba1 immunolabeling revealed dense microglial activation in both instances of TBM and CM, maximal in the proximity to meningeal inflammation. This probably reflects microglial response to signals emanating from the infective organisms by rapidly entering into the cell cycle and undergoing extensive expansion, manifesting as cellular hypertrophy and/or an increase in cell numbers [35-36].

Following bacterial infection, microglia change their morphology to an activated phenotype [37,38], rapidly up-regulate surface receptors of immune regulation such as complement receptors and major histocompatibility complex (MHC) class I and II molecules [33,39], and transform into phagocytic macrophages [35]. The present study recorded numerous MHC-II molecules expressing microglial cells and systemic marrow-derived monocytes present in close proximity to subarachnoid exudate inflammation as well as in perivascular areas. These cells are the main antigen-presenting cells of the CNS, playing an important role in immune modulation in the brain.

Several comparative studies conducted on TBM with and without associated HIV concluded that HIV infection does not significantly alter the neurological morbidity of tuberculous meningitis [40-42]. However, these studies were mainly confined to the clinical evaluation, CSF abnormalities, and neuroimaging. In the present pathomorphological study, we observed that in HIV-negative TBM cases, higher numbers of activated amoeboid microglia were found to home around the cortical neurons in the grey matter in contrast to the HIV-positive TBM cases (low area fraction of Iba1-labeled cells). This feature was especially evident in the proximity of subarachnoid inflammation and surface parenchymal granulomas, possibly attempting to insulate them from incoming afferent input, a process known as synaptic stripping $[43,44]$. This suggests that microglial activation and intervention can lead to cortical deafferentation, resulting in variable degrees of cognitive deficits.
Dense microglial proliferation and activation was recorded adjacent to meningeal inflammation in the hippocampus, affecting the white matter of the alvius and perforant pathway, which in turn could affect hippocampal function. Dense microglial activation, the majority of which was in the macrophagic stage around the granuloma, was a feature in HIV-negative TBM cases, whereas granuloma formation was lacking in HIV-positive TBM brains. Earlier studies have reported that co-infection of HIV with TBM enhances the pathology by functional disruption of local immune response within the granuloma [45-47].

In line with our observations in cryptococcal meningitis cases, Goldman et al. [48] reported that inflammation in the meninges is associated with subpial and subependymal glial activation, enhanced CD4 expression in microglia, and the presence of perivascular HLA-DR (MHC-II) and HAM-56 positive cells, highlighting activation of perivascular microglia modulating the recruitment and immune activation of inflammatory cells from systemic circulation. More importantly, MHC-II-positive perivascular microglial cells are reported to be critical for host resistance to Cryptoccocus neoformans [49]. Earlier studies have shown that nitric oxide (NO) production by human microglia is not sufficient for phagocytosis and clearing of cryptococci [50,51], though it can limit cryptococcal growth [52]. Furthermore, Lee et al. [53] revealed that microglia can ingest and inhibit the growth of Cryptococcus neoformans, and the ingested yeast forms eventually lyse the microglial cells. The present study showed highly activated microglia surrounding parenchymal cryptococcal cystic lesions, with expression of MHCII as well as macrophagic marker (HAM-56). These features were more evident in HIV-negative CM cases than in HIV-positive CM cases. This suggests that microglial activation and its capability to produce NO may get suppressed when associated with HIV and the resultant immunosuppression. The higher number of HLA-DR-positive cells in perivascular locations in both grey and white matter in the brains of HIVpositive CM cases supports the hypothesis of Aguirre and Miller [49] that MHC-II-positive microglial cells are more efficient in mediating resistance to Cryptococcus neoformans than are MHC-II-negative parenchymal microglia.

This study recorded higher cell counts of microglia in cases of -HIV-positive and HIV-negative TBM cases than in controls and cases of HIV encephalitis without opportunistic infections. However, the microglial cell number between HIV-positive and 
HIV-negative TBM cases was not significantly different, reflecting a relatively similar degree of microglial activation and proliferation. In contrast to the cell counts, the percent area occupied by Iba1immunostained cells was lower in HIV-negative TBM cases, which had higher numbers of amoeboid microglia with retracted processes, whereas in HIVpositive TBM cases, microglial cells displayed numerous, branched, and long processes and low activation features. In addition to self-renewal by entering into the cell cycle, activated microglial cells also secrete various chemokines such as MCP-1 and RANTES, which facilitate recruitment of peripheral macrophages into the brain parenchyma in viral infections [54]. Both microglial proliferation and peripheral macrophage cell recruitment could play a major role in increasing cells of macrophage lineage following infection.

In the present study, variable degrees of astroglial and microglial apoptosis were detected in both TBM and CM. This glial apoptosis in infective conditions reflects the down-regulation of activated glial cells and their subsequent elimination. Microglia are reported to undergo autoregulation-induced apoptosis [55]. The possible reason for microglial loss in cases with opportunistic infections might be to eliminate the activated microglial cells, thus preventing accumulation of over-activated harmful microglial cells from the milieu [55-57]. Alternatively, damaged or inflamed neurons during infections send signals to activate microglia to provide support to them by enhancing the production of cytokines and growth factors. If the neurons survive, possibly the excess number of activated microglia are eliminated by programmed cell death in due course. On the other hand, if neurons are not able to survive, the acute neuronal death leads to the transformation of microglia into macrophages to clear out the debris. A higher number of amoeboid macrophagic microglia in cases of TBM without HIV cases reflects the possibility that microglia are transformed into macrophages to clear the debris of dead neurons. On the other hand, if damaged neurons continue to send signals to microglia, the chronic microglial over-activation leads to degenerative changes and senescence in cases of chronic infection $[56,58]$. Our observations showed perineuronal microglial cells undergoing apoptosis in TBM and CM, supporting the above hypothesis. After this type of apoptotic microglial elimination, the neurons lose their microglial support, probably progressing to slow neurodegeneration.
The topographic distribution of apoptotic neurons in the CNS of HIV-infected individuals is closely associated with macrophage/microglial activation, suggesting that factors released from activated macrophages/microglia are the source of neurotoxic compounds leading to neuronal dysfunction. Several studies have suggested that HIV-infected macrophages/microglia express elevated levels of noxious factors implicated in neurotoxicity including but not limited to, proinflammatory cytokines (TNFalpha, IL-1beta, IL-6), chemokines (CCL4, CCL5), nitric oxide, and excitatory amino acids (glutamate, Lcysteine) $[59,60]$. Therefore, these findings suggest that HIV infection in the CNS promotes neuronal injury and/or neurotoxicity through the release of soluble factors produced by infected macrophages/microglia and astroglial cells.

In the present study, astrocytes showed expression of caspase-3 in both HIV-positive and HIV-negative TBM and CM cases. On the other hand, infectionassociated neuronal apoptosis was considered rare $[61,62]$. It has been described earlier that exposure to a limited insult can protect the cells by preconditioning them, by a process of activation of caspase- 3 binding to caspase-binding proteins, which can consequently prevent caspase activation, thus retarding apoptosis [63]. Such a mechanism may play a role in the glial changes as observed in the present study following mycobacterial and cryptococcal infection with or without associated HIV infection.

The presence of beaded astrocytic processes in TBM and CM cases (more prominently in the HIVnegative group) and the absence of it in the brains of cases of HIV not associated with opportunistic infections reflects the induced degenerating/dystrophic changes in the astrocytes $[64,65]$. This could be due to exposure of astrocytes to hypoxia following infectionassociated vascular changes similar to the pathology described by Zhang and Stone in the retina [66]. Many astrocytes with this degenerative appearance were shown to express caspase-3. The death of astrocytes and the loss of their support mechanisms would significantly compromise surrounding neurons and may be the main reason for neurodegenerative mechanisms following chronic inflammation.

HIV-1 infection is also characterized by demyelination and white matter damage [67,68]. Our study showed caspase-3 labeled oligodendrocytes in the white matter of brains of HIV-infected individuals along with opportunistic infections, along with demyelination as revealed by LFB staining. This 
damage to fiber tracts in the brain could accentuate the pathology and enhance clinical sequels.

\section{Conclusions}

To summarize, Mycobacterium tuberculosis appears to trigger both microgliosis and astrogliosis more extensively than does cryptococcal meningitis. The presence of increased astroglial and microglial responses is relevant to the neurobiological mechanisms involved in the pathogenesis of these infections, because both microglia and astrocytes are important for neuronal-glial interactions and synaptic function. Microglial and astroglial activation seems to play a major role in the neuroimmune mechanisms of disease in the CNS because these cells are part of the first line of defense of the innate immune system of the CNS and contribute to the modulation of immune responses by producing both proinflammatory and anti-inflammatory cytokines. The location of activated astroglial and microglia mainly near meningeal exudate reflects their roles in immune defense. The microglial and astroglial response in the CNS may have a dichotomous role in the inflammatory responses of the brain as a direct effector of infectionrelated damage and, on the other hand, as a neuroprotectant.

\section{Acknowledgements}

The work was supported by a grant from the Department of Biotechnology, India. Special thanks to the support from Human Brain Tissue Repository, NIMHANS, Bangalore, India. The provision of facilities by the Bioinformatics Centre, Jiwaji University, Gwalior, India, is also gratefully acknowledged.

\section{References}

1. Tan IL, Smith BR, von Geldern G, Mateen FJ, McArthur JC (2011) HIV-associated opportunistic infections of the CNS. Lancet Neurol 11: 605-617.

2. Joint United Nations Programme on HIV/AIDS (UNAIDS) (2010) Global report: UNAIDS report on the global AIDS epidemic 2010. Geneva: UNAIDS. Available: http://www.unaids.org/globalreport/Global_report.htm. Accessed 28 November 2012.

3. d'Arminio Monforte A, Cinque P, Mocroft A, Goebel FD, Antunes F, Katlama C, Stenz Justesen U, Vella S, Kirk O, Lundgren J (2004) Changing incidence of central nervous system diseases in the EuroSIDA cohort. Ann Neurol 55: 320-328.

4. Garvey L, Winston A, Walsh J, Post F, Porter K, Gazzard B, Fisher M, Leen C, Pillay D, Hill T (2011) HIV-associated central nervous system diseases in the recent combination antiretroviral therapy era. Eur J Neurol 18: 527-534.
5. Kwan CK, Ernst JD (2011) HIV and tuberculosis: a deadly human syndemic. Clin Microbiol Rev 24: 351-376.

6. Rich AR, McCordock HA (1933) The pathogenesis of tuberculous meningitis. Bull Johns Hopkins Hosp 52: 2-37.

7. Jarvis JN, Lawn SD, Vogt M, Bangani N, Wood R, Harrison TS (2009) Screening for cryptococcal antigenemia in patients accessing an antiretroviral treatment program in South Africa. Clin Infect Dis 48: 856-862.

8. Mahadevan A, Shankar SK, Satishchandra P, Ranga U, Chickabasaviah YT, Santosh V, Vasanthapuram R, Pardo CA, Nath A, Zink MC (2007) Characterization of human immunodeficiency virus (HIV)-infected cells in infiltrates associated with CNS opportunistic infections in patients with HIV clade C infection. J Neuropathol Exp Neurol 66: 799808.

9. Shankar SK, Mahadevan A, Satishchandra P, Uday Kumar R, Yasha TC, Santosh V, Shankar Chandramuki A, Ravi V, Nath A (2005) Neuropathology of HIV/AIDS with an overview of the Indian scene. Indian J Med Res 121: 468-488.

10. Rock RB, Hu S, Gekker G, Sheng WS, May B, Kapur V, Peterson PK (2005) Mycobacterium tuberculosis-induced cytokine and chemokine expression by human microglia and astrocytes: effects of dexamethasone. J Infect Dis 192: 20542058.

11. Lee YC, Wang JT, Sun HY, Chen YC (2011) Comparisons of clinical features and mortality of cryptococcal meningitis between patients with and without human immunodeficiency virus infection. J Microbiol Immunol Infect 44: 338-345.

12. Liao CH, Chi CY, Wang YJ, Tseng SW, Chou CH, Ho CM, Lin PC, Ho MW, Wang JH (2012) Different presentations and outcomes between HIV-infected and HIV-uninfected patients with Cryptococcal meningitis. J Microbiol Immunol Infect 45: 296-304.

13. van Elsland SL, Springer P, Steenhuis IHM, van Toorn R, Schoeman JF, van Furth AM (2011) Tuberculous Meningitis: Barriers to Adherence in Home Treatment of Children and Caretaker Perceptions. J Trop Pediatr 58: 275-279.

14. Siddappa NB, Dash PK, Mahadevan A, Jayasuryan N, Hu F, Dice B, Keefe R, Satish KS, Satish B, Sreekanthan K (2004) Identification of subtype $\mathrm{C}$ human immunodeficiency virus type 1 by subtype-specific PCR and its use in the characterization of viruses circulating in the southern parts of India. J Clin Microbiol 42: 2742-2751.

15. Bignami A, Eng LF, Dahl D, Uyeda CT (1972) Localization of the glial fibrillary acidic protein in astrocytes by immunofluorescence. Brain Res 43: 429-435.

16. Su JH, Nichol KE, Sitch T, Sheu P, Chubb C, Miller BL, Cotman CW (2000) DNA damage and activated caspase-3 expression in neurons and astrocytes: evidence for apoptosis in frontotemporal dementia. Exp Neurol 163: 9-19.

17. Streit WJ, Braak H, Xue QS, Bechmann I (2009) Dystrophic (senescent) rather than activated microglial cells are associated with tau pathology and likely precede neurodegeneration in Alzheimer's disease. Acta Neuropathol 118: 475-485.

18. Kettenmann H, Verkhratsky A (2008) Neuroglia: the 150 years after. Trends Neurosci 31: 653.

19. Raivich G, Jones LL, Werner A, Blothmann H, Doetschmann T, Kreutzberg GW (1999) Molecular signals for glial activation: pro-and anti-inflammatory cytokines in the injured brain. Acta Neurochir Suppl 73: 21-30. 
20. Sofroniew MV (2009) Molecular dissection of reactive astrogliosis and glial scar formation. Trends Neurosci 32: 638-647.

21. Sofroniew MV, Vinters HV (2010) Astrocytes: biology and pathology. Acta Neuropathol 119: 7-35.

22. $\mathrm{Hu} \mathrm{J}$, Ferreira A, Van Eldik LJ (1997) S100 $\beta$ induces neuronal cell death through nitric oxide release from astrocytes. J Neurochem 69: 2294-2301.

23. Patro N, Shrivastava M, Tripathi S, Patro IK (2009) S100 $\beta$ upregulation: a possible mechanism of deltamethrin toxicity and motor coordination deficits. Neurotoxicol Teratol 31: 169-176.

24. Van Eldik LJ, Wainwright MS (2003) The Janus face of glialderived S100B: beneficial and detrimental functions in the brain. Restor Neurol Neurosci 21: 97-108.

25. Huttunen HJ, Kuja-Panula J, Sorci G, Agneletti AL, Donato R, Rauvala H (2000) Coregulation of neurite outgrowth and cell survival by amphoterin and S100 proteins through receptor for advanced glycation end products (RAGE) activation. J Biol Chem 275: 40096-40105.

26. Li Y, Wang J, Sheng JG, Liu L, Barger SW, Jones RA, Van Eldik LJ, Mrak RE, Griffin WST (1998) S100 $\beta$ Increases Levels of $\beta$-Amyloid Precursor Protein and Its Encoding mRNA in Rat Neuronal Cultures. J Neurochem 71: 14211428.

27. Rickmann M, Wolff JR (1995) S100 protein expression in subpopulations of neurons of rat brain. Neuroscience 67: 977991.

28. Petrova TV, Hu J, Van Eldik LJ (2000) Modulation of glial activation by astrocyte-derived protein S100B: differential responses of astrocyte and microglial cultures. Brain Res 853: 74-80.

29. Esposito G, De Filippis D, Cirillo C, Sarnelli G, Cuomo R, Iuvone $\mathrm{T}$ (2006) The astroglial-derived $\mathrm{S} 100 \beta$ protein stimulates the expression of nitric oxide synthase in rodent macrophages through p38 MAP kinase activation. Life Sci 78: 2707-2715.

30. Anders K, Steinsapir KD, Iverson DJ, Glasgow BJ, Layfield LJ, Brown WJ, Cancilla PA, Verity MA, Vinters HV (1986) Neuropathologic findings in the acquired immunodeficiency syndrome (AIDS). Clin Neuropath 5: 1.

31. Berger JR, Harris JO, Gregoriost J, Norenbergt M (1990) Cerebrovascular disease in AIDS: a case-control study. AIDS 4: 239

32. Davalos D, Grutzendler J, Yang G, Kim JV, Zuo Y, Jung S, Littman DR, Dustin ML, Gan WB (2005) ATP mediates rapid microglial response to local brain injury in vivo. Nat Neurosci 8: $752-758$.

33. Kreutzberg GW (1996) Microglia: a sensor for pathological events in the CNS. Trends Neurosci 19: 312-318.

34. Nimmerjahn A, Kirchhoff F, Helmchen F (2005) Resting microglial cells are highly dynamic surveillants of brain parenchyma in vivo. Science 308: 1314-1318.

35. Graeber MB, Streit WJ, Kreutzberg GW (1988) The microglial cytoskeleton: vimentin is localized within activated cellsin situ. J Neurocytol 17: 573-580.

36. Solomon JN, Lewis CAB, Ajami B, Corbel SY, Rossi F, Krieger C (2006) Origin and distribution of bone marrowderived cells in the central nervous system in a mouse model of amyotrophic lateral sclerosis. Glia 53: 744-753.

37. Djukic M, Mildner A, Schmidt H, Czesnik D, Bruck, W, Priller J, Nau R, Prinz M (2006) Circulating monocytes engraft in the brain, differentiate into microglia and contribute to the pathology following meningitis in mice. Brain 129: 2394-2403.

38. Patro IK, Amit, Shrivastava M, Bhumika S, Patro N (2010) Poly I:C induced microglial activation impairs motor activity in adult rats. Indian J Exp Biol 48: 104-109.

39. Kielian T (2004) Microglia and chemokines in infectious diseases of the nervous system: views and reviews. Front Biosci 9: 732-750.

40. Berenguer J, Moreno S, Laguna F, Vicente T, Adrados M, Ortega A, Gonzalez-LaHoz J, Bouza E (1992) Tuberculous meningitis in patients infected with the human immunodeficiency virus. N Engl J Med 326: 668-672.

41. Dube MP, Holtom PD, Larsen RA (1992) Tuberculous meningitis in patients with and without human immunodeficiency virus infection. Am J Med 93: 520-524.

42. Thwaites GE, Duc Bang N, Huy Dung N, Thi Quy H, Thi Tuong Oanh D, Thi Cam Thoa N, Quang Hien N, Tri Thuc N, Ngoc Hai N, Thi Ngoc Lan N, Ngoc Lan N, Hong Duc N, Ngoc Tuan V, Huu Hiep C, Thi Hong Chau T, Phuong Mai P, Thi Dung N, Stepniewska K, Simmons CP, White NJ, Tinh Hien T, Farrar JJ (2005) The influence of HIV infection on clinical presentation, response to treatment, and outcome in adults with tuberculous meningitis. J Infect Dis 192: 2134 2141.

43. Schiefer J, Kampe K, Dodt HU, Zieglgansberger W, Kreutzberg GW (1999) Microglial motility in the rat facial nucleus following peripheral axotomy. J Neurocytol 28: 439453.

44. Yamada K, Tanaka N, Nakanishi K, Kamei N, Ishikawa M, Mizuno T, Igarashi K, Ochi M (2008) Modulation of the secondary injury process after spinal cord injury in Bach1deficient mice by heme oxygenase-1. J Neurosurg Spine 9: 611-620.

45. Bezuidenhout J, Roberts T, Muller L, Van Helden P, Walzl G (2009) Pleural tuberculosis in patients with early HIV infection is associated with increased TNF-alpha expression and necrosis in granulomas. PLoS One 4: e4228.

46. de Noronha AL, Bafica A, Nogueira L, Barral A, Barral-Netto M (2008) Lung granulomas from Mycobacterium tuberculosis/HIV-1 co-infected patients display decreased in situ TNF production. Pathol Res Pract 204: 155.

47. Lawn SD, Butera ST, Shinnick TM (2002) Tuberculosis unleashed: the impact of human immunodeficiency virus infection on the host granulomatous response to Mycobacterium tuberculosis. Microbes Infect 4: 635-646.

48. Goldman DL, Khine H, Abadi J, Lindenberg DJ, Pirofski L, Niang R, Casadevall A (2001) Serologic evidence for Cryptococcus neoformansinfection in early childhood. Pediatrics 107: e66-e66.

49. Aguirre K, Miller S (2002) MHC class II-positive perivascular microglial cells mediate resistance to Cryptococcus neoformans brain infection. Glia 39: 184-188.

50. Chao CC, Gekker G, Hu S, Peterson PK (1994) Human microglial cell defense against Toxoplasma gondii. The role of cytokines. J Immunol 152: 1246-1252.

51. Peterson PK, Hu S, Anderson WR, Chao CC (1994) Nitric oxide production and neurotoxicity mediated by activated microglia from human versus mouse brain. J Infect Dis 170: 457-460.

52. Lipovsky MM, Gekker G, Hu S, Hoepelman AIM, Peterson PK (1998) Morphine Enhances Complement ReceptorMediated Phagocytosis of Cryptococcus neoformans by Human Microglia. Clin Immunol Immunopathol 87: 163-167. 
53. Lee SC, Kress Y, Zhao ML, Dickson DW, Casadevall A (1995) Cryptococcus neoformans survive and replicate in human microglia. Lab Invest 73: 871.

54. Eugenin EA, Berman JW (2003) Chemokine-dependent mechanisms of leukocyte trafficking across a model of the blood-brain barrier. Methods 29: 351-361.

55. Lee R, Kermani P, Teng KK, Hempstead BL (2001) Regulation of cell survival by secreted proneurotrophins. Science 294: 1945-1948.

56. Liu B, Wang K, Gao HM, Mandavilli B, Wang JY, Hong JS (2001) Molecular consequences of activated microglia in the brain: overactivation induces apoptosis. J Neurochem 77: 182-189.

57. Suk K, Lee J, Hur J, Kim YS, Lee MS, Cha S, Yeou Kim S, Kim H (2001) Activation-induced cell death of rat astrocytes. Brain Res 900: 342-347.

58. Polazzi E, Contestabile A (2006) Overactivation of LPSstimulated microglial cells by co-cultured neurons or neuronconditioned medium. J Neuroimmunol 172: 104-111.

59. Garden GA, Budd SL, Tsai E, Hanson L, Kaul M, D'Emilia DM, Friedlander RM, Yuan J, Masliah E, Lipton SA (2002) Caspase cascades in human immunodeficiency virusassociated neurodegeneration. J Neurosci 22: 4015-4024.

60. Kaul M, Garden GA, Lipton SA (2001) Pathways to neuronal injury and apoptosis in HIV-associated dementia. Nature 410: 988-994.

61. Atzori C, Ghetti B, Piva R, Srinivasan AN, Zolo P, Delisle MB, Mirra SS, Migheli A (2001) Activation of the JNK/p38 pathway occurs in diseases characterized by tau protein pathology and is related to tau phosphorylation but not to apoptosis. J Neuropathol Exp Neurol 60: 1190-1197.

62. Gleckman AM, Jiang Z, Liu Y, Smith TW (1999) Neuronal and glial DNA fragmentation in Pick's disease. Acta Neuropathol 98: 55-61.
63. McLaughlin BA, Hartnett KA, Erhardt JA, Legos JJ, White RF, Barone FC, Aizenman E (2003) Caspase 3 activation is essential for neuroprotection in preconditioning. Proc Natl Acad Sci USA 100: 715-720.

64. Broe M, Kril J, Halliday GM (2004) Astrocytic degeneration relates to the severity of disease in frontotemporal dementia. Brain 127: 2214-2220.

65. Martin JA, Craft DK, Su JH, Kim RC, Cotman CW (2001) Astrocytes degenerate in frontotemporal dementia: possible relation to hypoperfusion. Neurobiol Aging 22: 195-207.

66. Zhang Y, Stone J (1997) Role of astrocytes in the control of developing retinal vessels. Invest Ophthalmol Vis Sci 38: 1653-1666.

67. Anthony IC, Bell PJE (2008) The neuropathology of HIV/AIDS. Int Rev Psychiatry 20: 15-24.

68. Boisse L, Gill MJ, Power C (2008) HIV infection of the central nervous system: clinical features and neuropathogenesis. Neurol Clin 26: 799-819.

\section{Corresponding author}

Prof. Ishan Patro

Professor of Zoology/ Neuroscience

Jiwaji University, Gwalior-474011, India

Phone: + 917512442789

Mobile: + 919425110063

Email: ishanpatro@gmail.com

Conflict of interests: No conflict of interests is declared. 